\title{
Anelastic tidal dissipation in multi-layer planets
}

\author{
F. Remus ${ }^{1,2,3}$, S. Mathis ${ }^{3,4}$, J.-P. Zahn ${ }^{1}$, and V. Lainey ${ }^{2}$ \\ ${ }^{1}$ LUTH, Observatoire de Paris - CNRS - Université Paris Diderot, 5 place Jules Janssen, 92195 Meudon Cedex, France \\ e-mail: [francoise.remus; jean-paul.zahn]@obspm.fr \\ 2 IMCCE, Observatoire de Paris - UMR 8028 du CNRS - Université Pierre et Marie Curie, 77 avenue Denfert-Rochereau, \\ 75014 Paris, France \\ e-mail: lainey@imcce.fr \\ ${ }^{3}$ Laboratoire AIM Paris-Saclay, CEA/DSM - CNRS - Université Paris Diderot, IRFU/SAp Centre de Saclay, 91191 Gif-sur-Yvette, \\ France \\ e-mail: stephane.mathis@cea.fr \\ ${ }^{4}$ LESIA, Observatoire de Paris - CNRS - Université Paris Diderot - Université Pierre et Marie Curie, 5 place Jules Janssen, \\ 92195 Meudon, France
}

Received 6 December 2011 / Accepted 24 February 2012

\begin{abstract}
Context. Earth-like planets have viscoelastic mantles, whereas giant planets may have viscoelastic cores. The tidal dissipation of these solid regions, which are gravitationally perturbed by a companion body, strongly depends on their rheology and the tidal frequency. Therefore, modeling tidal interactions provides constraints on planets' properties and helps us to understand their history and evolution, in either our solar system or exoplanetary systems.

Aims. We examine the equilibrium tide in the anelastic parts of a planet for every rheology, and by taking into account the presence of a fluid envelope of constant density. We show how to obtain the different Love numbers describing its tidal deformation, and discuss how the tidal dissipation in the solid parts depends on the planet's internal structure and rheology. Finally, we show how our results may be implemented to describe the dynamical evolution of planetary systems.

Methods. We expand in Fourier series the tidal potential exerted by a point mass companion, and express the dynamical equations in the orbital reference frame. The results are cast in the form of a complex disturbing function, which may be implemented directly in the equations governing the dynamical evolution of the system.

Results. The first manifestation of the tide is to distort the shape of the planet adiabatically along the line of centers. The response potential of the body to the tidal potential then defines the complex Love numbers, whose real part corresponds to the purely adiabatic elastic deformation and the imaginary part accounts for dissipation. The tidal kinetic energy is dissipated into heat by means of anelastic friction, which is modeled here by the imaginary part of the complex shear modulus. This dissipation is responsible for the imaginary part of the disturbing function, which is implemented in the dynamical evolution equations, from which we derive the characteristic evolution times.

Conclusions. The rate at which the system evolves depends on the physical properties of the tidal dissipation, and specifically on (1) how the shear modulus varies with tidal frequency, (2) the radius, and (3) the rheological properties of the solid core. The quantification of the tidal dissipation in the solid core of giant planets reveals a possible high dissipation that may compete with dissipation in fluid layers.
\end{abstract}

Key words. planets and satellites: general - planets and satellites: physical evolution - planets and satellites: individual: Jupiter planets and satellites: dynamical evolution and stability - planets and satellites: individual: Saturn - planet-star interactions

\section{Introduction and general context}

Since 1995, a large number of extrasolar planets have been discovered, which have displayed a wide range of physical parameters (Santos et al. 2007). The question has arisen quite naturally of their habitability, i.e. whether they could allow the development of life. Determining factors are the presence of liquid water and a protective magnetic field, properties that are closely linked to the values of the rotational and orbital elements of the planetary systems. These elements strongly depend on the action of tides. Once a planetary system is formed in a turbulent accretion disk, its fate is determined by the initial conditions and the mass ratio of the planet to its hosting star. Through tidal interaction between components, the system evolves either to a stable state of minimum energy (where all spins are aligned, the orbits are circular, and the rotation of each body is synchronized with the orbital motion) or the companion tends to spiral into the parent body. By converting kinetic energy into heat by means of internal friction, tidal interactions modify the orbital and rotational properties of the components of the considered system, and thus their structure through internal heating. This mechanism depends sensitively on the internal structure and dynamics of the perturbed body.

Studies have been carried out on the tidal effects in fluid bodies such as stars and envelopes of giant planets (Ogilvie \& Lin 2004-2007; Ogilvie 2009; Remus et al. 2012). However, the planetary solid regions, such as the mantles of Earth-like planets or the rocky cores of giant planets, when present (e.g. Guillot 1999; Gaulme et al. 2011), may also contribute to tidal dissipation. The first study of a tidally deformed elastic body was done by Lord Kelvin (1863), who applied it to an incompressible homogeneous Earth. Further developments were made by Love (1911), who introduced a set of dimensionless numbers, the so-called Love numbers, to quantify the tidal perturbation. 
More recently, Greff-Lefftz (2005) generalized these results to the case of a spheroidal rotating Earth. As for Dermott (1979), he considered a two-layer model and studied the impact of a tidally deformed static fluid shell on the deformation of an elastic solid core.

If the body is not perfectly elastic, i.e. if its internal structure is anelastic, the tidal deformation presents a lag with respect to the tension exerted by the tidal force, and causes the dissipation of kinetic energy. Several studies have addressed this problem of tidal dissipation using linear viscoelastic models. Peale \& Cassen (1978) evaluated the tidal dissipation in the Moon by considering various models of internal structure. Tobie et al. (2005) applied the Maxwell rheological model to evaluate the dissipation in Titan and Europa; Ross \& Schubert (1986) compared three different linear models of viscoelasticity (KelvinVoigt, Maxwell, and standard anelastic solid), to which Henning et al. (2009) added the Burgers body. All these studies have inspired our interest in the tidal dissipation resulting from the anelastic deformation of the solid parts of a planet when perturbed by a companion.

We study here the tidal dissipation in a planet that possesses an anelastic core consisting of a mix of ice and rock, surrounded by a fluid envelope, such as an ocean. The planet is part of a binary system where what we call the companion (or perturber) may be either the hosting star or a satellite of the planet. Owing to the tide exerted by the companion, the core of the two-layer planet is deformed elastically, but because of the anelasticity of the material composing the core, this deformation is accompanied by a viscous dissipation that we evaluate for every rheology. As an illustration, we present our results for a Maxwell body. We then compare the value of the tidal dissipation in the presence of a fluid envelope with that achieved by the fully solid planet, and examine the dependence of the results on the relative sizes of the core and the planet, the relative densities, and the viscoelastic parameters. In the last section, we establish the equations governing the dynamical evolution of the system, from which we deduce the characteristic times of circularization, synchronization, and spin alignments.

\section{Elastic deformations of a solid body under tidal perturbation}

\subsection{The system}

We consider a planet $A$ of mass $M_{A}$, consisting of a rocky (or icy) core and a fluid envelope, rotating at the angular velocity $\Omega$ and tidally perturbed by a point-like body of mass $M_{B}$ moving around $A$ on a Keplerian orbit, of semi-major axis $a$ and eccentricity $e$, at the mean motion $n$. We locate any point $M$ in space by its usual spherical coordinates $(r, \theta, \varphi)$ in a spin equatorial reference frame $\mathcal{R}_{\mathrm{E}}:\left\{A, \boldsymbol{X}_{\mathrm{E}}, \boldsymbol{Y}_{\mathrm{E}}, \boldsymbol{Z}_{\mathrm{E}}\right\}$ centered on body $A$ and whose axis $\left(A, Z_{\mathrm{E}}\right)$ has the direction of the rotation axis of $A$. The corresponding configuration is illustrated in Fig. 1.

In Sect. 2, we first assume that planet $A$ has no fluid layer and its internal structure is supposed to be perfectly elastic. We then denote by $\rho$ its density and $R$ its mean radius.

\subsection{The tidal potential}

The planet is submitted to a tidal force exerted by the perturber $B$, which derives from a perturbing time-dependent potential $U(\boldsymbol{r}, t)$. Following Zahn $(1966 \mathrm{a}, \mathrm{b})$ and generalizing it by using Kaula (1962), Lambeck (1980), Yoder (1995-1997), and Mathis \& Le Poncin (2009, hereafter MLP09) in the studied case

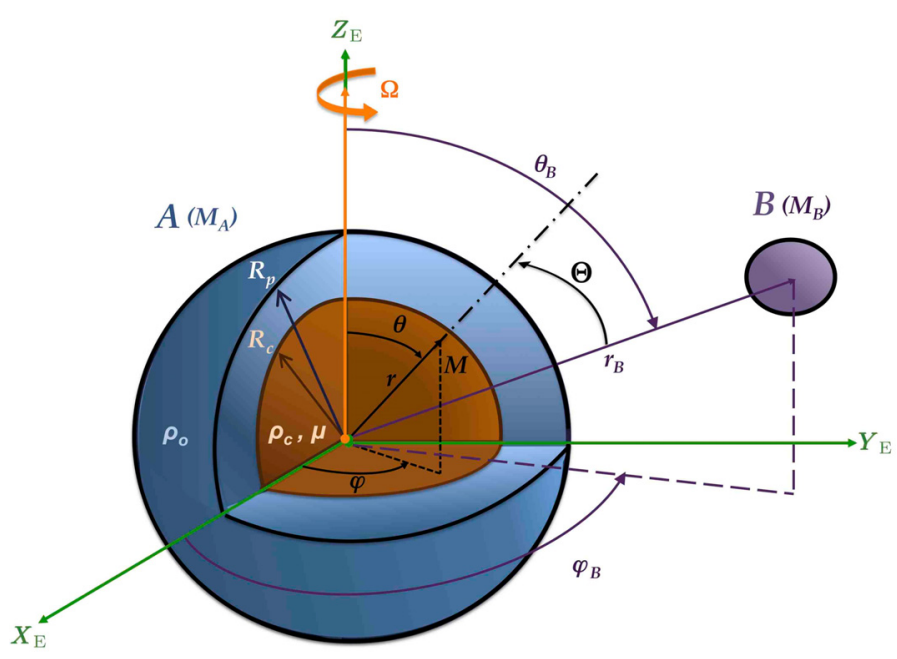

Fig. 1. Spherical coordinates system attached to the equatorial reference frame $\mathcal{R}_{\mathrm{E}}:\left\{A, \boldsymbol{X}_{\mathrm{E}}, \boldsymbol{Y}_{\mathrm{E}}, \boldsymbol{Z}_{\mathrm{E}}\right\}$ associated with body $A$. A point $M$ is located by its position vector $\boldsymbol{r} \equiv(r, \theta, \varphi)$; the point mass body $B$ by $\boldsymbol{r}_{B} \equiv\left(r_{B}, \theta_{B}, \varphi_{B}\right)$

of a close binary system where spins are not aligned, the components are not synchronized with the orbital motion, and the orbit is not circular, we expand the tidal potential $U$ in spherical harmonics $Y_{l}^{m}(\theta, \varphi)$ in $\mathcal{R}_{\mathrm{E}}$.

Before we proceed, we need to define the Euler angles that link both the spin equatorial frame $\mathcal{R}_{\mathrm{E}}:\left\{A, \boldsymbol{X}_{\mathrm{E}}, \boldsymbol{Y}_{\mathrm{E}}, \boldsymbol{Z}_{\mathrm{E}}\right\}$ of the central body $A$ and the orbital frame $\mathcal{R}_{O}:\left\{A, \boldsymbol{X}_{\mathrm{O}}, \boldsymbol{Y}_{\mathrm{O}}, \boldsymbol{Z}_{\mathrm{O}}\right\}$ to the quasi-inertial frame $\mathcal{R}_{\mathrm{R}}:\left\{A, \boldsymbol{X}_{\mathrm{R}}, \boldsymbol{Y}_{\mathrm{R}}, \boldsymbol{Z}_{\mathrm{R}}\right\}$ whose axis $\boldsymbol{Z}_{\mathrm{R}}$ has the direction of the total angular momentum of the whole system.

We need the three following Euler angles to locate the orbital reference frame $\mathcal{R}_{\mathrm{O}}$ with respect to $\mathcal{R}_{\mathrm{R}}$ :

- $I$, the inclination of the orbital plane of $B$;

- $\omega^{*}$, the argument of the orbit pericenter;

- $\Omega^{*}$, the longitude of the orbit ascending node.

The equatorial reference frame $\mathcal{R}_{\mathrm{E}}$ is defined by three other Euler angles with respect to $\mathcal{R}_{\mathrm{R}}$ :

- $\varepsilon$, the obliquity of the rotation axis of $A$;

- $\Theta^{*}$, the mean sideral angle defined by $\Omega=\mathrm{d} \Theta^{*} / \mathrm{d} t$;

- $\phi^{*}$, the general precession angle.

We refer to Fig. 2 for an illustration of the relative position of these three reference frames and their associated angles. For convenience, all our following developments are made in the spin equatorial frame $\mathcal{R}_{\mathrm{E}}$ of $A$ (as in MLP09).

All of our following results are derived from the Kaula's transform (Kaula 1962), which is used to explicitly express the whole generic multipole expansion in spherical harmonics of the perturbing potential $U$ in terms of the Keplerian orbital elements of $B$ in the equatorial $A$-frame

$$
\begin{aligned}
\frac{1}{r_{B}^{l+1}} P_{l}^{m}\left(\cos \theta_{B}\right) \mathrm{e}^{\mathrm{i} m \varphi_{B}}= & \frac{1}{a^{l+1}} \sum_{j=-l}^{l} \sum_{p=0}^{l} \sum_{q \in \mathbb{Z}}\left\{\sqrt{\frac{2 l+1}{4 \pi} \frac{(l-|j|) !}{(l+|j|) !}}\right. \\
& \left.\times d_{j, m}^{l}(\varepsilon) F_{l, j, p}(I) G_{l, p, q}(e) \mathrm{e}^{\mathrm{i} \Psi_{l, m, j, p, q}}\right\}
\end{aligned}
$$




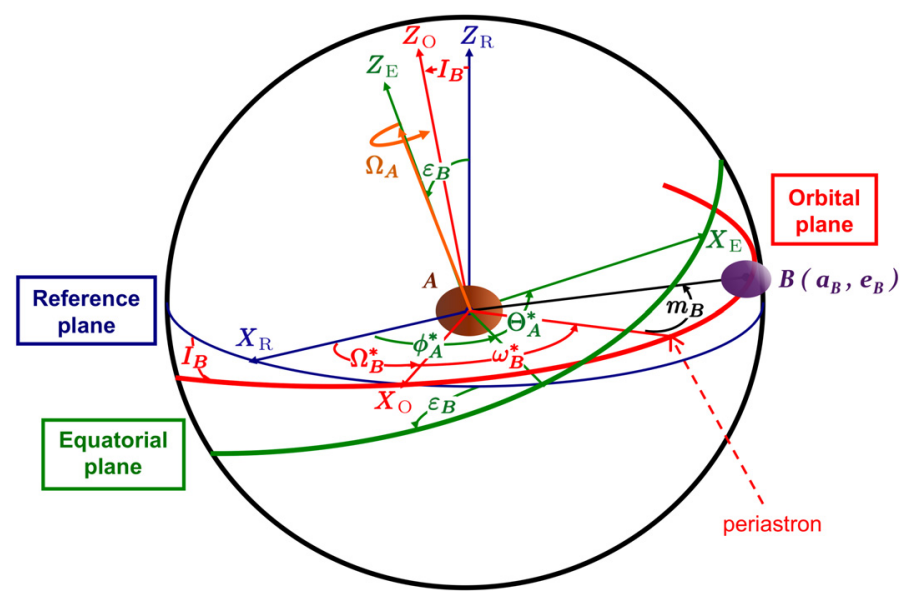

Fig. 2. Inertial reference $\left(\mathcal{R}_{\mathrm{R}}\right)$, orbital $\left(\mathcal{R}_{\mathrm{O}}\right)$, and equatorial $\left(\mathcal{R}_{\mathrm{E}}\right)$ rotating frames, and associated Eulers angles of orientation.

where $\theta_{B}$ and $\varphi_{B}$ are respectively the colatitude and the longitude of the point mass perturber $B$, and the phase argument is given by

$\Psi_{l, m, j, p, q}(t)=\sigma_{l, m, p, q}(n, \Omega) t+\tau_{l, m, j, p, q}\left(\omega^{*}, \Omega^{*}, \phi^{*}\right)$.

We defined here the tidal frequency

$\sigma_{l, m, p, q}(n, \Omega)=(l-2 p+q) n-m \Omega$,

and the phase

$\tau_{l, m, j, p, q}=(l-2 p) \omega^{*}+j\left(\Omega^{*}-\phi^{*}\right)+(l-m) \frac{\pi}{2}$.

We consider binary systems that are close enough for the tidal interaction to play a role, but also where the companion is far away (or small) enough to be treated as a point mass. We are then allowed to assume the quadrupolar approximation, where we only keep the first mode of the potential, $l=2$

$$
\begin{aligned}
U(r, \theta, \varphi, t)= & \operatorname{Re}\left\{\sum_{m=-2}^{2} \sum_{j=-2}^{2} \sum_{p=0}^{2} \sum_{q \in \mathbb{Z}}\right. \\
& \left.\times U_{m, j, p, q}(r) P_{2}^{m}(\cos \theta) \mathrm{e}^{\mathrm{i} \Phi_{2, m, j, p, q}(\varphi, t)}\right\}
\end{aligned}
$$

where

$\Phi_{2, m, j, p, q}(\varphi, t)=m \varphi-\Psi_{2, m, j, p, q}(t)$.

The functions $U_{m, j, p, q}(r, \theta)$ may be expressed in terms of the Keplerian elements (the semi-major axis $a$ of the orbit, its eccentricity $e$, and its inclination $I$ ) and the obliquity $\varepsilon$ of the rotation axis of $A$, as

$$
\begin{aligned}
U_{m, j, p, q}(r)= & (-1)^{m} \sqrt{\frac{(2-m) !(2-|j|) !}{(2+m) !(2+|j|) !}} \\
& \times \frac{\mathcal{G} M_{B}}{a^{3}}\left[d_{j, m}^{2}(\varepsilon) F_{2, j, p}(I) G_{2, p, q}(e)\right] r^{2},
\end{aligned}
$$

where $\mathcal{G}$ is the gravitational constant.

The obliquity function $d_{j, m}^{2}(\gamma)$ is defined, for $j \geqslant m$, by

$$
\begin{aligned}
d_{j, m}^{2}(\gamma)= & (-1)^{j-m}\left[\frac{(2+j) !(2-j) !}{(2+m) !(2-m) !}\right]^{\frac{1}{2}} \\
& \times\left[\cos \left(\frac{\gamma}{2}\right)\right]^{j+m}\left[\sin \left(\frac{\gamma}{2}\right)\right]^{j-m} \mathrm{P}_{2-j}^{(j-m, j+m)}(\cos \gamma),
\end{aligned}
$$

Table 1. Values of the obliquity function $d_{j, m}^{2}(\varepsilon)$ in the case where $j \geqslant m$ obtained from Eq. (8) (from MLP09).

\begin{tabular}{ccc}
\hline \hline$j$ & $m$ & $d_{j, m}^{2}(\varepsilon)$ \\
\hline 2 & 2 & $\left(\cos \frac{\varepsilon}{2}\right)^{4}$ \\
2 & 1 & $-2\left(\cos \frac{\varepsilon}{2}\right)^{3}\left(\sin \frac{\varepsilon}{2}\right)$ \\
2 & 0 & $\sqrt{6}\left(\cos \frac{\varepsilon}{2}\right)^{2}\left(\sin \frac{\varepsilon}{2}\right)^{2}$ \\
1 & 1 & $\left(\cos \frac{\varepsilon}{2}\right)^{4}-3\left(\cos \frac{\varepsilon}{2}\right)^{2}\left(\sin \frac{\varepsilon}{2}\right)^{2}$ \\
1 & 0 & $-\sqrt{6} \cos \varepsilon\left(\cos \frac{\varepsilon}{2}\right)\left(\sin \frac{\varepsilon}{2}\right)$ \\
0 & 0 & $1-6\left(\cos \frac{\varepsilon}{2}\right)^{2}\left(\sin \frac{\varepsilon}{2}\right)^{2}$ \\
\hline
\end{tabular}

Table 2. Values of the inclination function $F_{2, j, p}(I)$ (from MLP09).

\begin{tabular}{ccc}
\hline \hline$j$ & $p$ & $F_{2, j, p}(I)$ \\
\hline 0 & 0 & $\frac{3}{8} \sin ^{2} I$ \\
0 & 1 & $-\frac{3}{4} \sin ^{2} I+\frac{1}{2}$ \\
0 & 2 & $\frac{3}{8} \sin ^{2} I$ \\
1 & 0 & $\frac{3}{4} \sin I(1+\cos I)$ \\
1 & 1 & $-\frac{3}{2} \sin I \cos I$ \\
1 & 2 & $-\frac{3}{4} \sin I(1-\cos I)$ \\
2 & 0 & $\frac{3}{4}(1+\cos I)^{2}$ \\
2 & 1 & $\frac{3}{2} \sin ^{2} I$ \\
2 & 2 & $\frac{3}{4}(1-\cos I)^{2}$ \\
\hline
\end{tabular}

Notes. Values for indices $j<0$ can be deduced from Eq. (10).

Table 3. Values of the eccentricity function $G_{2, p, q}(e)$ (from MLP09).

\begin{tabular}{cc|cc|c}
\hline \hline$p$ & $q$ & $p$ & $q$ & $G_{2, p, q}(e)$ \\
\hline 0 & -2 & 2 & 2 & 0 \\
0 & -1 & 2 & 1 & $-\frac{1}{2} \mathrm{e}+\cdots$ \\
0 & 0 & 2 & 0 & $1-\frac{5}{2} \mathrm{e}^{2}+\cdots$ \\
0 & 1 & 2 & -1 & $\frac{7}{2} \mathrm{e}+\cdots$ \\
0 & 2 & 2 & -2 & $\frac{17}{2} \mathrm{e}^{2}+\cdots$ \\
1 & -2 & 1 & 2 & $\frac{9}{4} \mathrm{e}^{2}+\cdots$ \\
1 & -1 & 1 & 1 & $\frac{3}{2} \mathrm{e}+\cdots$ \\
& & 1 & 0 & $\left(1-\mathrm{e}^{2}\right)^{-3 / 2}$ \\
\hline
\end{tabular}

where $\mathrm{P}_{l}^{\alpha, \beta}(x)$ are the Jacobi polynomials (cf. MLP09). The values of these functions, for indices $j<m$, are deduced from $d_{j, m}^{2}(\pi+\gamma)=(-1)^{2-j} d_{-j, m}^{2}(\gamma)$ or from their symmetry properties: $d_{j, m}^{2}(\gamma)=(-1)^{j-m} d_{-j,-m}^{2}(\gamma)=d_{m, j}^{2}(-\gamma)$; moreover, we know that $d_{j, m}^{2}(0)=\delta_{j, m}$. Values are given in Table 1 .

We also define, the inclination function $F_{2, j, p}(I)$

$$
\begin{aligned}
F_{2, j, p}(I)= & (-1)^{j} \frac{(2+j) !}{4 p !(2-p) !}\left[\cos \left(\frac{I}{2}\right)\right]^{j-2 p+2}\left[\sin \left(\frac{I}{2}\right)\right]^{j+2 p-2} \\
& \times \mathrm{P}_{2-j}^{(j+2 p-2, j-2 p+2)}(\cos I)
\end{aligned}
$$

with the symmetry property

$F_{2,-j, p}(I)=\left[(-1)^{2-j} \frac{(2-j) !}{(2+j) !}\right] F_{2, j, p}(I)$.

Values are given in Table 2.

The eccentricity functions $G_{2, p, q}(e)$ are polynomial functions of $\mathrm{e}^{q}$ (see Kaula 1962). Their values for the usual sets $\{2, p, q\}$ are given in Table 3, where we know that in the case of weakly eccentric orbits, the summation over a small number of values of $q$ is sufficiently accurate $(q \in \mathbb{L}-2,2 \rrbracket)$. In the following, we 
denote by $\mathbb{I}=\llbracket-2,2 \rrbracket \times \llbracket-2,2 \rrbracket \times \llbracket 0,2 \rrbracket \times \mathbb{Z}$ the set in which the quadruple $\{m, j, p, q\}$ takes its values.

If we simplify the expansion of the potential to the case where spins are aligned and are perpendicular to the orbital plan, where obliquity $\varepsilon$ and orbital inclination $I$ are zero, Eq. (5) reduces to the expression of the potential given by Zahn (1977).

The tidal force induces a displacement of each particle constituting the planet, thus causing some deformations. In particular, the core's surface is deformed as described by the Love theory (Love 1911).

\subsection{Dynamical equations for a solid body and their boundary conditions}

To describe the internal evolution of the main component $A$ submitted to the perturbations induced by the tidal potential presented above, we use the Eulerian formalism (Dahlen et al. 1999). The system of equations, needed to follow the motion of a particle, is composed by the Eulerian momentum (11a) and mass (11b) conservation laws, and the Poisson Eq. (11c) satisfied by the potential $\Phi$ of self-gravitation

$\rho \frac{\partial^{2} \boldsymbol{s}}{\partial t^{2}}=\boldsymbol{\nabla} \cdot \overline{\overline{\boldsymbol{\sigma}}}+\rho \boldsymbol{\nabla}(\Phi+U)$,

$\frac{\partial \rho}{\partial t}+\nabla \cdot\left(\rho \frac{\partial s}{\partial t}\right)=0$

$\nabla^{2} \Phi=-4 \pi G \rho$,

where $s$ designates the displacement vector and $\overline{\bar{\sigma}}$ is the stress tensor. We complete this system with the constitutive equation linking the stress exerted on the body to the resulting deformation. Assuming that the tidal effect corresponds to a traction applied to the body, with no rotational contribution, the deformation tensor reduces to the strain tensor $\overline{\overline{\boldsymbol{\epsilon}}}$

$\overline{\bar{\epsilon}}=\frac{1}{2}\left[\nabla s+(\nabla s)^{\mathrm{T}}\right]$,

where $\overline{\overline{\boldsymbol{h}}}^{\mathrm{T}}$ designates the transposed tensor of $\overline{\overline{\boldsymbol{h}}}$. We then get a relation linking the stress tensor $\overline{\bar{\sigma}}$ to the strain tensor $\overline{\overline{\boldsymbol{\epsilon}}}$ that accounts for the rheology of the body, and that we represent by a function $\mathcal{F}_{\text {rh }}$

$\overline{\overline{\boldsymbol{\sigma}}}=\mathcal{F}_{\mathrm{rh}}(\overline{\overline{\boldsymbol{\epsilon}}})$.

To solve this system of Eq. (11), we need to apply boundary conditions to the five previous equations, assuming that there is neither a displacement (Eq. (12a)) nor an attraction (Eq. (12b)) at the center of mass $r=0$, that the gravitational potential is continuous (Eq. (12c)), and that the Lagrangian traction vanishes (12d) at the surface $r=R$ such that

$$
\begin{aligned}
& \left.\boldsymbol{s}\right|_{r=0}=\mathbf{0}, \\
& \left.(\Phi+U)\right|_{r=0}=\mathbf{0}, \\
& {[\Phi]_{R^{-}}^{R^{+}}=0, \quad \text { i.e.: } \quad\left[\frac{\partial \Phi}{\partial r}+4 \pi \mathcal{G} \rho s_{r}\right]_{R^{-}}^{R^{+}}=0,} \\
& \left.\left(\boldsymbol{e}_{r} \cdot \overline{\overline{\boldsymbol{\sigma}}}\right)\right|_{r=R}=0 .
\end{aligned}
$$

\subsection{Linearization of the system}

Assuming that tidal effects, and thus the resulting elastic deformation, are small amplitude perturbations to the hydrostatic equilbrium, we are allowed to linearize the system of Eq. (11) and its boundary conditions in Eq. (12). To do so, we expand a scalar quantity $X$ as

$X(r, \theta, \varphi, t)=X_{0}(r)+X^{\prime}(r, \theta, \varphi, t)$

where $X_{0}$ designates the spherically symmetrical profile of $X$, and $X^{\prime}$ represents the perturbation due to the tidal potential. The displacement $s$ and the tidal potential $U$ are also considered as perturbations. Thus, to first order in $\|s\|$, we obtain the following form of the system of Eq. (11):

$$
\begin{aligned}
\rho_{0} \frac{\partial^{2} \boldsymbol{s}}{\partial t^{2}} & =\boldsymbol{\nabla} \cdot \overline{\overline{\boldsymbol{\sigma}}}+\rho_{0} \boldsymbol{\nabla}\left(\Phi^{\prime}+U\right)+\rho^{\prime} \boldsymbol{\nabla} \Phi_{0}, \\
\rho^{\prime}+\boldsymbol{\nabla} \cdot\left(\rho_{0} \boldsymbol{s}\right) & =0, \\
\boldsymbol{\nabla}^{2} \Phi^{\prime} & =-4 \pi \mathcal{G} \rho^{\prime}, \\
\overline{\overline{\boldsymbol{\sigma}}} & =\left(K-\frac{2}{3} \mu\right) \operatorname{tr}(\overline{\overline{\boldsymbol{\epsilon}}}) \overline{\overline{\boldsymbol{I}}}+2 \mu \overline{\overline{\boldsymbol{\epsilon}}},
\end{aligned}
$$

where we have made use of Hooke's law in Eq. (14d), which is a linear constitutive law that governs elastic materials as long as the load does not exceed the material's elastic limit, in the case of an isotropic material (i.e. whose properties are independent of direction in space). This means that the strain is directly proportional to stress, through the bulk modulus $K$ and the shear modulus $\mu$. The reference state, drawn from an up-to-date planetary structure model, is governed by the Poisson and static momentum equations

$$
\begin{aligned}
\nabla^{2} \Phi_{0} & =-4 \pi G \rho_{0}, \\
\nabla P_{0} & =\rho_{0} \nabla \Phi_{0},
\end{aligned}
$$

where we have made use of the convention for the gravity $\boldsymbol{g}_{0}=$ $\nabla \Phi_{0}$.

\subsection{Analytical solutions for a homogeneous incompressible body}

To solve the linear system of Eq. (14), we expand all scalar quantities in spherical harmonics $Y_{l}^{m}(\theta, \varphi)$. Moreover, as all vectorial quantities that intervene in Eqs. (14a), (14b) are poloidal, we may expand them in the vectorial spherical harmonics basis $\left[\boldsymbol{R}_{l}^{m}(\theta, \varphi), \boldsymbol{S}_{l}^{m}(\theta, \varphi)\right]$, where $\boldsymbol{R}$ refers to the radial part and $\boldsymbol{S}$ to the spheroidal part of a given vector (Rieutord 1987; Mathis \& Zahn 2005)

$\forall(l, m) \in \mathbb{N} \times \mathbb{L}-l, l \rrbracket,\left\{\begin{array}{l}\boldsymbol{R}_{l}^{m}(\theta, \varphi)=Y_{l}^{m}(\theta, \varphi) \boldsymbol{e}_{r}, \\ \boldsymbol{S}_{l}^{m}(\theta, \varphi)=\boldsymbol{\nabla}_{\mathrm{S}}\left[Y_{l}^{m}(\theta, \varphi)\right],\end{array}\right.$

where $\boldsymbol{\nabla}_{\mathrm{S}}$ designates the horizontal gradient

$\boldsymbol{\nabla}_{\mathrm{S}}=\frac{\partial \cdot}{\partial \theta} \boldsymbol{e}_{\theta}+\frac{1}{\sin \theta} \frac{\partial \cdot}{\partial \varphi} \boldsymbol{e}_{\varphi}$

We introduce six radial functions $y_{\{1 \ldots 6\}}^{m}(r)$ (Takeuchi \& Saito 1972) to expand all quantities in spherical harmonics, at the quadrupolar approximation $(l=2)$, namely

- the displacement

$$
\begin{aligned}
\boldsymbol{s}(r, \theta, \varphi, t)= & \sum_{(m, j, p, q) \in \mathbb{I}}\left[y_{1}^{m}(r) \boldsymbol{R}_{2}^{m}(\theta, \varphi)\right. \\
& \left.+y_{3}^{m}(r) \boldsymbol{S}_{2}^{m}(\theta, \varphi)\right] \mathrm{e}^{\mathrm{i} \Psi_{2, m, j, p, q}(t)} ;
\end{aligned}
$$


- the total potential

$$
\left(U+\Phi^{\prime}\right)(r, \theta, \varphi, t)=\sum_{(m, j, p, q) \in \mathbb{I}} y_{5}^{m}(r) Y_{2}^{m}(\theta, \varphi) \mathrm{e}^{\mathrm{i} \Psi_{2, m, j, p, q}(t)}
$$

- the Lagrangian traction

$$
\begin{aligned}
\boldsymbol{e}_{r} \cdot \overline{\overline{\boldsymbol{\sigma}}}(r, \theta, \varphi, t)= & \sum_{(m, j, p, q) \in \mathbb{I}}\left[y_{2}^{m}(r) \boldsymbol{R}_{2}^{m}(\theta, \varphi)\right. \\
& \left.+y_{4}^{m}(r) \boldsymbol{S}_{2}^{m}(\theta, \varphi)\right] \mathrm{e}^{\mathrm{i} \Psi_{2, m, j, p, q}(t)} ;
\end{aligned}
$$

- the Lagrangian attraction (introduced to express the continuity of the gradient of the potential)

$$
\forall m \in \mathbb{I}-2,2 \rrbracket, y_{6}^{m}(r)=\frac{\mathrm{d}}{\mathrm{d} r}\left[y_{5}^{m}(r)\right]-4 \pi \mathcal{G} \rho_{0} y_{1}^{m}(r) .
$$

The linear system governing the radial functions $y_{\{1 \ldots 6\}}^{m}(r)$ is given in Appendix. In the case of an incompressible $(K \rightarrow+\infty)$ and homogeneous body ( $\mu, \rho_{0}=$ const.), the system of Eq.(14) constrained by boundary conditions in Eq. (12) admits the solutions, based on the expansions in Eq. (18): $\forall m \in \mathbb{I}-2,2 \rrbracket$,

$$
\begin{aligned}
& y_{1}^{m}(r)= \sum_{j, p, q} \frac{k_{2}}{r R g_{s}}\left(\frac{8}{3} R^{2}-r^{2}\right) U_{m, j, p, q}(r), \\
& y_{2}^{m}(r)=\sum_{j, p, q}\left[2 \mu \frac{k_{2}}{r^{2} R g_{s}}\left(\frac{8}{3} R^{2}+\frac{1}{2} r^{2}\right)\right. \\
&+\frac{4}{3} \pi G \rho^{2} \frac{k_{2}}{R g_{s}}\left(\frac{8}{3} R^{2}-\frac{1}{2} r^{2}\right) \\
&\left.\quad-\rho\left(1+k_{2}\right)\right] U_{m, j, p, q}(r), \\
& y_{3}^{m}(r)=\sum_{j, p, q} \frac{k_{2}}{r R g_{s}}\left(\frac{4}{3} R^{2}-\frac{5}{6} r^{2}\right) U_{m, j, p, q}(r), \\
& y_{4}^{m}(r)=\sum_{j, p, q} \frac{8 \mu}{3} \frac{k_{2}}{r R g_{s}}\left(R^{2}-r^{2}\right) U_{m, j, p, q}(r), \\
& y_{5}^{m}(r)=\sum_{j, p, q}\left(1+k_{2}\right) U_{m, j, p, q}(r), \\
& y_{6}^{m}(r)=\sum_{j, p, q}\left[\frac{2\left(1+k_{2}\right)}{r}\right. \\
&\left.+4 \pi G \rho_{0} \frac{k_{2}}{r R g_{s}}\left(r^{2}-\frac{8}{3} R^{2}\right)\right] U_{m, j, p, q}(r),
\end{aligned}
$$

where we have introduced the acceleration of gravity at the surface $g_{s}$ and the second-order Love number $k_{2}$. The latter compares the perturbed part $\Phi^{\prime}(R)$ of the self-gravitational potential at the surface of a fully-solid planet of mean radius $R$, deformed by tidal force, with the tidal perturbing potential $U(R)$

$k_{2} \stackrel{\text { def }}{=} \frac{\Phi^{\prime}(R)}{U(R)}$

The expression of $k_{2}$ is established in Sect. 3, for an ocean-free planet (Eq. (61)) or a two-layer planet (Eq. (63)).

\section{Modified elastic tidal theory in presence of a fluid envelope}

We now assume that planet $A$ is not entirely solid, but has a static fluid envelope. We follow the method proposed by
Dermott (1979) to evaluate how the anelastic dissipation is modified by the presence of a fluid layer surrounding the solid region. The first step consists in determining the behavior of the elastic response in this configuration. We denote by $R_{\mathrm{c}}$ (respectively (resp.) $R_{\mathrm{p}}$ ) the mean radius of the solid core (resp. of the whole planet, including the height of the fluid layer) while $\rho_{\mathrm{c}}$ and $\rho_{\mathrm{o}}$ designate the density of the core and the ocean respectively, which are both assumed to be uniform, as a first step. More generally, all quantities are written with a " $c$ " subscript when evaluated at the core boundary and with a " $p$ " subscript if taken at the surface of the planet. The evolution of the system is described in the orbital frame $\mathcal{R}_{\mathrm{O}}:\left\{A, \boldsymbol{X}_{\mathbf{O}}, \boldsymbol{Y}_{\mathbf{O}}, \boldsymbol{Z}_{\mathbf{O}}\right\}$ centered on $A$ and comoving with the perturber $B$. We use polar coordinates $(r, \Theta)$ to locate a point $P$, where $r$ is the distance to the center of $A$, and $\Theta$ is the angle formed by the radial vector and the line of centers.

\subsection{Vertical deformation at the boundary of the core}

In $\mathcal{R}_{\mathrm{O}}$, the tidal potential takes the form (Dermott 1979)

$U(\boldsymbol{r})=-\zeta(r) g(r) P_{2}(\cos \Theta)=-\zeta_{\mathrm{c}} g_{\mathrm{c}} \frac{r^{2}}{R_{\mathrm{c}}^{2}} P_{2}(\cos \Theta)$,

where we have introduced the tidal height

$\zeta(r)=\frac{M_{B}}{M(r)}\left(\frac{r}{a}\right)^{3} r$

and the gravity

$g(r)=\frac{G M(r)}{r^{2}}$,

where $M(r)$ is the fraction of mass of the planet inside the radius $r$.

The expression of the tidal potential in the rotating frame of $B$ (Eq. (21)) is linked to its expression in the equatorial inertial frame (Eq. (5)), through the Kaula's transform (Eq. (1)). The Legendre polynomial summation formula

$$
\begin{aligned}
P_{2}(\cos \Theta)= & \mathcal{R e}\left[\sum_{m=0}^{2} \frac{(2-m) !}{(2+m) !}\left(2-\delta_{0, m}\right) P_{2}^{m}(\cos \theta) \mathrm{e}^{\mathrm{i} m \varphi}\right. \\
& \left.\times P_{2}^{m}\left(\cos \theta_{B}\right) \mathrm{e}^{-\mathrm{i} m \varphi_{B}}\right]
\end{aligned}
$$

involves the term $P_{2}^{m}\left(\cos \theta_{B}\right) \mathrm{e}^{-\mathrm{i} m \varphi_{B}}$ in Eq. (21), which has to be transformed following Eqs. (1), (2) to obtain Eq. (5).

In this section, we are interested in the modication of Love numbers caused by the presence of a fluid envelope on top of the solid core. Thus, we focus on the deformations of the core's surface, particularly the vertical displacements. Love (1911) proved that tidal deformations could be described by the same harmonic function as the tidal potential causing it. Therefore, the equations of the core and planet boundaries are respectively of the form

$r_{\mathrm{c}} \equiv R_{\mathrm{c}}+s_{r}\left(R_{\mathrm{c}}\right)=R_{\mathrm{c}}\left[1+S_{2} P_{2}(\cos \Theta)\right]$,

$r_{\mathrm{p}}=R_{\mathrm{p}}\left[1+T_{2} P_{2}(\cos \Theta)\right]$.

Thus, $s_{r}\left(R_{\mathrm{c}}\right)=R_{\mathrm{c}} S_{2} P_{2}(\cos \Theta)$ represents the radial displacement at the core's boundary corresponding to the vertical tidal deformation of amplitude $S_{2} P_{2}(\cos \Theta)$. In 1909, Love defined the number $h_{2}$, as the ratio of the amplitude of the vertical displacement at the surface of the planet to the equilibrium tidal height (the disturbing potential divided by the undisturbed surface gravity, both taken at the surface of the core) in the case of 

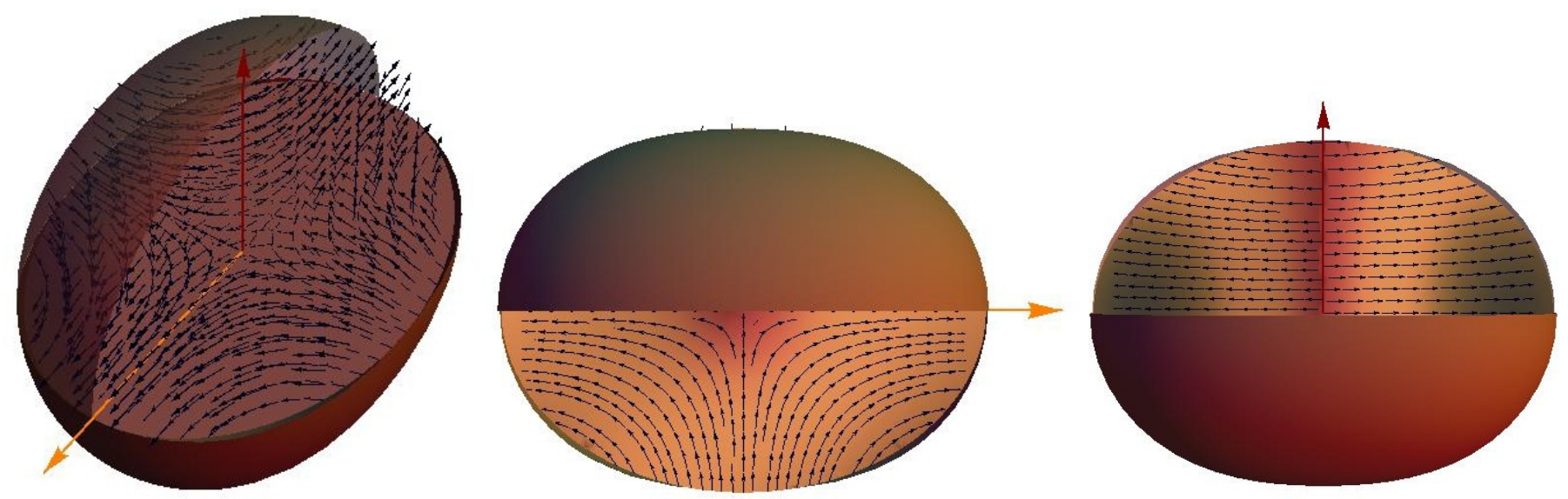

Fig. 3. Left: tidal displacement $s$. Middle: equatorial slice of $s$. Right: meridional slice of $s$. The orange arrow indicates the direction of the perturber $B$, and the red one corresponds to the rotation axis of $A$. The two slices are planes of symmetry.

a fully-solid planet. Solving the whole system of equations, he determined its expression as

$h_{2} \stackrel{\text { def }}{=} \frac{s_{r}\left(R_{\mathrm{c}}\right)}{U\left(R_{\mathrm{c}}\right) / g_{\mathrm{c}}} \equiv \frac{R_{\mathrm{c}} S_{2}}{\zeta_{\mathrm{c}}}=\frac{5}{2} \frac{1}{1+\bar{\mu}}$

where $\bar{\mu}$ is called the effective rigidity, in the sense that it evaluates the relative importance of elastic and gravitational forces as

$\bar{\mu}=\frac{19 \mu}{2 \rho_{\mathrm{c}} g R_{\mathrm{c}}}$

In the presence of the fluid envelope, the ratio of the amplitude of the tidal surface vertical displacement to the tidal height is modulated by a multiplicative factor $F$, owing to the additional loading exerted by the tidally deformed fluid layer. We may then introduce a new notation $h_{2}^{F}$ for the modified Love number in the presence of a fluid envelope

$h_{2}^{F} \stackrel{\text { def }}{=} \frac{s_{r}\left(R_{\mathrm{c}}\right)}{U\left(R_{\mathrm{c}}\right) / g_{\mathrm{c}}} \equiv \frac{R_{\mathrm{c}} S_{2}}{\zeta_{\mathrm{c}}}=F h_{2}=F \times \frac{5 / 2}{1+\bar{\mu}}$.

We have now to express this factor as a function of the parameters of the system. To do so, we have to list all the forces acting on the surface of the core. Before carrying out the study of these forces, we introduce a specific notation. All physical quantities $X(\boldsymbol{r})$ are separated into two terms: the first corresponds to the constant part that does not depend on where the quantity is calculated; the second one, called the "effective deforming" contribution and denoted $X^{\prime}(\boldsymbol{r})$ ), is a term proportional to the spherical surface harmonic $P_{2}$ (see Eq. (24)).

\subsection{Gravitational forces acting on the surface of the core}

The planet is not only subjected to the direct action of the tidal potential $U$, but also to the self-gravitational potential $\Phi$ perturbed by the first. In calculating the latter, we have to consider the contributions of both the solid core and the fluid envelope $\Phi_{c}$ and $\Phi_{\mathrm{o}}$, respectively.

At any point $\boldsymbol{r}$ of the core, $\Phi_{\mathrm{c}}(\boldsymbol{r})$ corresponds to the internal potential created by the core

$\Phi_{\mathrm{c}}(\boldsymbol{r})=-\frac{g_{\mathrm{c}}}{R_{\mathrm{c}}}\left(\frac{3 R_{\mathrm{c}}^{2}-r^{2}}{2}+\frac{3}{5} r^{2} S_{2} P_{2}\right)$.
At the same point $\boldsymbol{r}, \Phi_{\mathrm{o}}(\boldsymbol{r})$ is the internal potential created by the fluid shell of density $\rho_{\mathrm{o}}$ and of lower and upper surface boundaries $r_{\mathrm{c}}$ and $r_{\mathrm{p}}$ respectively:

$$
\begin{aligned}
\Phi_{\mathrm{o}}(\boldsymbol{r})= & -\frac{\rho_{\mathrm{o}}}{\rho_{\mathrm{c}}} \frac{g_{\mathrm{c}}}{R_{\mathrm{c}}}\left[\left(\frac{3 R_{\mathrm{p}}^{2}-r^{2}}{2}+\frac{3}{5} r^{2} T_{2} P_{2}\right)\right. \\
& \left.-\left(\frac{3 R_{\mathrm{c}}^{2}-r^{2}}{2}+\frac{3}{5} r^{2} S_{2} P_{2}\right)\right] .
\end{aligned}
$$

Therefore, at any point $\boldsymbol{r}$ inside the core, $V(\boldsymbol{r})=U(\boldsymbol{r})+\Phi_{\mathrm{c}}(\boldsymbol{r})+$ $\Phi_{\mathrm{o}}(\boldsymbol{r})$ has the following expression

$V(\boldsymbol{r})=-\frac{1}{2} \frac{g_{\mathrm{c}}}{R_{\mathrm{c}}}\left[-r^{2}+3 R_{\mathrm{c}}^{2}\left(1-\frac{\rho_{\mathrm{o}}}{\rho_{\mathrm{c}}}+\frac{\rho_{\mathrm{o}}}{\rho_{\mathrm{c}}} \frac{R_{\mathrm{p}}^{2}}{R_{\mathrm{c}}^{2}}\right)\right]-V^{\prime}(\boldsymbol{r})$,

where the effective deforming potential is expressed by

$V^{\prime}(\boldsymbol{r})=-Z r^{2} P_{2}$,

where $Z$ is a constant that depends on the characteristics of the planet

$Z=\frac{g_{\mathrm{c}}}{R_{\mathrm{c}}}\left[\frac{\zeta_{\mathrm{c}}}{R_{\mathrm{c}}}+\frac{3}{5} \frac{\rho_{\mathrm{o}}}{\rho_{\mathrm{c}}}\left(T_{2}-S_{2}\right)+\frac{3}{5} S_{2}\right]$.

We then obtain its expression, which is correct to first order in $S_{2} P_{2}$ or $T_{2} P_{2}$, at any point $\boldsymbol{r}_{\mathrm{c}}=r_{\mathrm{c}} \boldsymbol{e}_{\mathrm{r}}$ of the surface of the core:

$V\left(\boldsymbol{r}_{\mathrm{c}}\right)=-g_{\mathrm{c}} R_{\mathrm{c}}\left[\left(1-\frac{3}{2} \frac{\rho_{\mathrm{o}}}{\rho_{\mathrm{c}}}\right)+\frac{3}{2} \frac{\rho_{\mathrm{o}}}{\rho_{\mathrm{c}}} \frac{R_{\mathrm{p}}^{2}}{R_{\mathrm{c}}^{2}}\right]+V^{\prime}\left(\boldsymbol{r}_{\mathrm{c}}\right)$,

where

$V^{\prime}\left(\boldsymbol{r}_{\mathrm{c}}\right)=-Z_{\mathrm{c}} R_{\mathrm{c}}^{2} P_{2}$,

and $Z_{\mathrm{c}}$ is a constant that depends on the characteristics of the planet:

$Z_{\mathrm{c}}=\frac{g_{\mathrm{c}}}{R_{\mathrm{c}}}\left[\frac{\zeta_{\mathrm{c}}}{R_{\mathrm{c}}}+\frac{3}{5} \frac{\rho_{\mathrm{o}}}{\rho_{\mathrm{c}}}\left(T_{2}-S_{2}\right)-\frac{2}{5} S_{2}\right]$.

Chree (1896) showed that the deformation of the core's surface under the gravitational forces, that derive from the effective deforming potential $V^{\prime}$, is the same as the deformation that would result from the outward normal traction $f_{1}^{T_{N}}$ applied at its mean surface $r=R_{\mathrm{c}}$

$f_{1}^{T_{\mathrm{N}}}\left(R_{\mathrm{c}}\right)=\rho_{\mathrm{c}} Z R_{\mathrm{c}}^{2} P_{2}$. 


\subsection{Total effective normal traction acting on the surface of the core}

The mean surface of the core is subjected to two additional forces induced by both the loading of the core and the loading of the ocean which is tidally deformed.

First, the pressure due to the differential overloading of the deformed elastoviscous matter on the mean surface of radius $R_{\mathrm{c}}$ is given by the product of the local gravity $g_{\mathrm{c}}$, the density of the core $\rho_{\mathrm{c}}$, and the solid tidal height $R_{\mathrm{c}} S_{2}$

$f_{2}^{T_{N}}\left(R_{\mathrm{c}}\right)=-\rho_{\mathrm{c}} g_{\mathrm{c}} R_{\mathrm{c}} S_{2} P_{2}$.

We also have to take into account the oceanic hydrostatic pressure. Following Zahn (1966a,b) and Remus et al. (2012), we express all scalar quantities $X(r, \Theta)$ as the sum of their spherically symmetrical profile $X^{0}(r)$ and their perturbation $X^{\prime}(r, \Theta)$ due to the tidal potential $U(r, \Theta) \propto P_{2}(\cos \Theta)$

$X(r, \Theta)=X^{0}(r)+X^{\prime}(r, \Theta) \equiv X^{0}(r)+\hat{X}(r) P_{2}(\cos \Theta)$.

The perturbations of pressure $P^{\prime}(r, \theta)$ obey the relation of hydrostatic equilibrium, which is to first order in $P_{2}(\cos \Theta)$ :

$\nabla P^{\prime}=\rho_{\mathrm{o}} \nabla V^{\prime}+\rho_{\mathrm{o}}^{\prime} \nabla V^{0}$.

Therefore, the $\Theta$-projection of (36) leads to

$P^{\prime}(r, \Theta)=\rho_{\mathrm{o}} V^{\prime}(r, \Theta)$.

Finally, since only the variable part of the pressure, i.e. $P^{\prime}$, contributes to the normal effective traction $f_{3}^{T_{N}}$ acting on the mean surface of the core, this latter takes the following expression

$f_{3}^{T_{N}}\left(R_{\mathrm{c}}\right)=P^{\prime}\left(\boldsymbol{r}_{\mathrm{c}}\right)=\rho_{\mathrm{o}} V^{\prime}\left(\boldsymbol{r}_{\mathrm{c}}\right)=-\rho_{\mathrm{o}} Z_{\mathrm{c}} R_{\mathrm{c}}^{2} P_{2}$.

The sum of these three forces, represented in Fig. 4, corresponds to the total normal effective traction $f^{T_{N}}\left(R_{\mathrm{c}}\right)=f_{1}^{T_{N}}\left(R_{\mathrm{c}}\right)+$ $f_{2}^{T_{N}}\left(R_{\mathrm{c}}\right)+f_{3}^{T_{N}}\left(R_{\mathrm{c}}\right)$ that deforms the mean surface of the core. Using Eqs. (33), (34), (38), we get

$f^{T_{N}}\left(R_{\mathrm{c}}\right)=\left(\rho_{\mathrm{c}} Z-\rho_{\mathrm{o}} Z_{\mathrm{c}}\right) R_{\mathrm{c}}^{2} P_{2}-\rho_{\mathrm{c}} g_{\mathrm{c}} R_{\mathrm{c}} S_{2} P_{2}$,

where the expressions of $Z$ and $Z_{\mathrm{c}}$ are given by Eqs. (31c) and $(32 \mathrm{c})$ respectively, so that

$f^{T_{N}}\left(R_{\mathrm{c}}\right)=X P_{2}(\cos \Theta)$,

where we have denoted by $X$ the quantity

$X=\frac{2}{5} \rho_{\mathrm{c}} g_{\mathrm{c}} R_{\mathrm{c}}\left(1-\frac{\rho_{\mathrm{o}}}{\rho_{\mathrm{c}}}\right)\left[\frac{5}{2} \frac{\zeta_{\mathrm{c}}}{R_{\mathrm{c}}}-S_{2}+\frac{3}{2} \frac{\rho_{\mathrm{o}}}{\rho_{\mathrm{c}}}\left(T_{2}-S_{2}\right)\right]$.

\subsection{Amplitude of the vertical deformation}

According to Melchior (1966), a deforming potential $\mathcal{U}_{2}$ of second order produces a deformation at each point $\boldsymbol{r}_{\mathrm{c}}$ of the surface of the core whose radial component takes the form

$\epsilon_{r r}=\frac{\left(8 R_{\mathrm{c}}^{2}-3 r_{\mathrm{c}}^{2}\right)}{19 \mu} \frac{\rho_{\mathrm{c}} \mathcal{U}_{2}}{r_{\mathrm{c}}^{2}}$.

To first order in $P_{2}$, recalling that $r_{\mathrm{c}}=R_{\mathrm{c}}\left(1+S_{2} P_{2}\right)$, this reduces to

$\epsilon_{r r}=\frac{5}{19 \mu} \rho_{\mathrm{c}} \mathcal{U}_{2}$

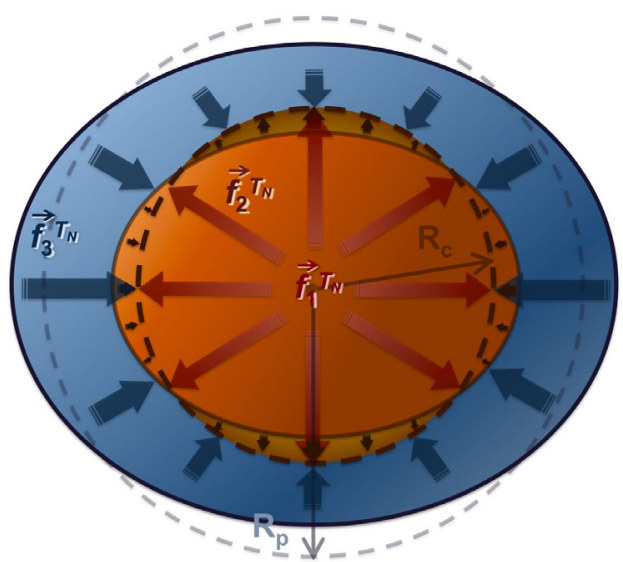

Fig. 4. Balance of forces acting on the mean surface of the core $r=R_{\mathrm{c}}$, where $f_{1}^{T_{N}}\left(R_{\mathrm{c}}\right)$ are the gravitational forces, $f_{2}^{T_{N}}\left(R_{\mathrm{c}}\right)$ the loading of the solid tide, and $f_{3}^{T_{N}}\left(R_{\mathrm{c}}\right)$ the hydrostatic pressure.

where $\rho_{\mathrm{c}} \mathcal{U}_{2}$ is the deforming traction $f^{T, N}\left(R_{\mathrm{c}}\right)=X P_{2}$ applied on the core. Furthermore, since the amplitude of the displacement is also given by $\epsilon_{r r}=S_{2} P_{2}$ (Eq. (25a)), we have the following equality

$S_{2} P_{2}=\frac{5}{19 \mu} X P_{2}$

Therefore the relation in Eq. (27) between $\mu$ and $\bar{\mu}$ and the expression in Eq. (41) for $X$ enable us to relate the deformation of the surfaces of the core $\left(S_{2}\right)$ to those of the ocean $\left(T_{2}\right)$ as

$S_{2}=\frac{1}{\bar{\mu}}\left(1-\frac{\rho_{\mathrm{o}}}{\rho_{\mathrm{c}}}\right)\left[\frac{5}{2} \frac{\zeta_{\mathrm{c}}}{R_{\mathrm{c}}}-S_{2}+\frac{3}{2} \frac{\rho_{\mathrm{o}}}{\rho_{\mathrm{c}}}\left(T_{2}-S_{2}\right)\right]$.

By definition, given in Eq. (28), the impedance $F$ is of the form

$F=\frac{2}{5}(1+\bar{\mu}) \frac{R_{\mathrm{c}}}{\zeta_{\mathrm{c}}} S_{2}$.

Since the surface of the planet is an equipotential, the total potential $V$ takes a constant value at any point $\boldsymbol{r}_{\mathrm{p}}$ of the surface of the ocean

$V\left(\boldsymbol{r}_{\mathrm{p}}\right) \equiv U\left(\boldsymbol{r}_{\mathrm{p}}\right)+\Phi\left(\boldsymbol{r}_{\mathrm{p}}\right)=$ const.

As $V-V^{\prime}=$ const. by definition, we get the simpler condition

$V^{\prime}\left(\boldsymbol{r}_{\mathrm{p}}\right) \equiv U\left(\boldsymbol{r}_{\mathrm{p}}\right)+\Phi^{\prime}\left(\boldsymbol{r}_{\mathrm{p}}\right)=$ const.

At a point $\boldsymbol{r}$ of the ocean, $\Phi_{\mathrm{c}}(\boldsymbol{r})$ corresponds to the external potential created by the core

$\Phi_{\mathrm{c}}(\boldsymbol{r})=-g_{\mathrm{c}} R_{\mathrm{c}}^{2}\left(\frac{1}{r}+\frac{3}{5} \frac{R_{\mathrm{c}}^{2} S_{2}}{r^{3}} P_{2}\right)$.

At the same point $\boldsymbol{r}, \Phi_{\mathrm{o}}(\boldsymbol{r})$ is the internal potential created by the fluid shell of density $\rho_{\mathrm{o}}$ and of lower and upper surface boundaries $r_{\mathrm{c}}$ and $r_{\mathrm{p}}$, respectively:

$$
\begin{aligned}
\Phi_{\mathrm{o}}(\boldsymbol{r})= & -g_{\mathrm{c}} \frac{R_{\mathrm{p}}^{3}}{R_{\mathrm{c}}} \frac{\rho_{\mathrm{o}}}{\rho_{\mathrm{c}}}\left(\frac{3 R_{\mathrm{p}}^{2}-r^{2}}{2 R_{\mathrm{p}}^{3}}+\frac{3}{5} \frac{r^{2} T_{2}}{R_{\mathrm{p}}^{3}} P_{2}\right) \\
& +g_{\mathrm{c}} R_{\mathrm{c}}^{2} \frac{\rho_{\mathrm{o}}}{\rho_{\mathrm{c}}}\left(\frac{1}{r}+\frac{3}{5} \frac{R_{\mathrm{c}}^{2} S_{2}}{r^{3}} P_{2}\right) .
\end{aligned}
$$


Therefore, $V(\boldsymbol{r})=U(\boldsymbol{r})+\Phi_{\mathrm{c}}(\boldsymbol{r})+\Phi_{\mathrm{o}}(\boldsymbol{r})$, at any point $\boldsymbol{r}$ inside the ocean, is given by

$V(\boldsymbol{r})=-g_{\mathrm{c}} R_{\mathrm{c}}^{2}\left[\left(1-\frac{\rho_{\mathrm{o}}}{\rho_{\mathrm{c}}}\right) \frac{1}{r}-\frac{\rho_{\mathrm{o}}}{\rho_{\mathrm{c}}} \frac{r^{2}}{2 R_{\mathrm{c}}^{3}}+\frac{3}{2} \frac{\rho_{\mathrm{o}}}{\rho_{\mathrm{c}}} \frac{R_{\mathrm{p}}^{2}}{R_{\mathrm{c}}^{3}}\right]+V^{\prime}(\boldsymbol{r})$,

where the effective deforming potential is expressed by

$V^{\prime}(\boldsymbol{r})=-g_{\mathrm{c}} R_{\mathrm{c}}^{2} W(r) P_{2}$,

$W$ being a function of the distance $r$ to the center of the planet

$W(r)=\frac{\zeta_{\mathrm{c}}}{R_{\mathrm{c}}} \frac{r^{2}}{R_{\mathrm{c}}^{3}}+\frac{3}{5} \frac{\rho_{\mathrm{o}}}{\rho_{\mathrm{c}}} \frac{r^{2}}{R_{\mathrm{c}}^{3}} T_{2}+\frac{3}{5}\left(1-\frac{\rho_{\mathrm{o}}}{\rho_{\mathrm{c}}}\right) \frac{R_{\mathrm{c}}^{2}}{r^{3}} S_{2}$.

We then obtain its expression at a point $\boldsymbol{r}_{\mathrm{p}}=r_{\mathrm{p}} \boldsymbol{e}_{r}$ of the surface of the planet

$V\left(\boldsymbol{r}_{\mathrm{p}}\right)=-g_{\mathrm{c}}\left[\left(1-\frac{\rho_{\mathrm{o}}}{\rho_{\mathrm{c}}}\right) \frac{R_{\mathrm{c}}^{2}}{R_{\mathrm{p}}}+\frac{\rho_{\mathrm{o}}}{\rho_{\mathrm{c}}} \frac{R_{\mathrm{p}}^{2}}{R_{\mathrm{c}}}\right]+V^{\prime}\left(\boldsymbol{r}_{\mathrm{p}}\right)$,

where

$V^{\prime}\left(\boldsymbol{r}_{\mathrm{p}}\right)=-g_{\mathrm{c}} W_{\mathrm{p}} P_{2}$,

$W_{\mathrm{p}}$ being a constant that depends on the planet's characteristics

$$
\begin{aligned}
W_{\mathrm{p}}= & \frac{R_{\mathrm{p}}^{2}}{R_{\mathrm{c}}^{2}} \zeta_{\mathrm{c}}+\left[\frac{3}{5}\left(1-\frac{\rho_{\mathrm{o}}}{\rho_{\mathrm{c}}}\right) \frac{R_{\mathrm{c}}^{4}}{R_{\mathrm{p}}^{3}}\right] S_{2} \\
& +\left[-\frac{2}{5} \frac{\rho_{\mathrm{o}}}{\rho_{\mathrm{c}}} \frac{R_{\mathrm{p}}^{2}}{R_{\mathrm{c}}}-\left(1-\frac{\rho_{\mathrm{o}}}{\rho_{\mathrm{c}}}\right) \frac{R_{\mathrm{c}}^{2}}{R_{\mathrm{p}}}\right] T_{2} .
\end{aligned}
$$

The condition in Eq. (48) then takes the form

$\frac{\zeta_{\mathrm{c}}}{R_{\mathrm{c}}}=-\frac{3}{5}\left(\frac{R_{\mathrm{c}}}{R_{\mathrm{p}}}\right)^{5}\left(1-\frac{\rho_{\mathrm{o}}}{\rho_{\mathrm{c}}}\right) S_{2}+\frac{2}{5} \frac{\rho_{\mathrm{o}}}{\rho_{\mathrm{c}}} \alpha T_{2}$,

where

$\alpha=1+\frac{5}{2} \frac{\rho_{\mathrm{c}}}{\rho_{\mathrm{o}}}\left(\frac{R_{\mathrm{c}}}{R_{\mathrm{p}}}\right)^{3}\left(1-\frac{\rho_{\mathrm{o}}}{\rho_{\mathrm{c}}}\right)$.

We can then eliminate the variable $T_{2}$ thanks to Eq. (45)

$$
\begin{aligned}
& \frac{2}{5} \frac{\rho_{\mathrm{o}}}{\rho_{\mathrm{c}}} \alpha T_{2}=\frac{\frac{2}{5} \alpha}{\left(\alpha+\frac{3}{2}\right)\left(1-\frac{\rho_{\mathrm{o}}}{\rho_{\mathrm{c}}}\right)} \\
& \times\left[1+\bar{\mu}-\frac{\rho_{\mathrm{o}}}{\rho_{\mathrm{c}}}+\frac{3}{2} \frac{\rho_{\mathrm{o}}}{\rho_{\mathrm{c}}}\left(1-\frac{\rho_{\mathrm{o}}}{\rho_{\mathrm{c}}}\right)+\frac{3}{2}\left(\frac{R_{\mathrm{c}}}{R_{\mathrm{p}}}\right)^{5}\left(1-\frac{\rho_{\mathrm{o}}}{\rho_{\mathrm{c}}}\right)^{2}\right] S_{2} .
\end{aligned}
$$

Inserting this relation into Eq. (53) for $\zeta_{\mathrm{c}} / R_{\mathrm{c}}$, and the resulting relation into Eq. (46) for $F$, we finally get

$$
F=\frac{\left(1-\frac{\rho_{\mathrm{o}}}{\rho_{\mathrm{c}}}\right)(1+\bar{\mu})\left(1+\frac{3}{2 \alpha}\right)}{1+\bar{\mu}-\frac{\rho_{\mathrm{o}}}{\rho_{\mathrm{c}}}+\frac{3}{2} \frac{\rho_{\mathrm{o}}}{\rho_{\mathrm{c}}}\left(1-\frac{\rho_{\mathrm{o}}}{\rho_{\mathrm{c}}}\right)-\frac{9}{4 \alpha}\left(\frac{R_{\mathrm{c}}}{R_{\mathrm{p}}}\right)^{5}\left(1-\frac{\rho_{\mathrm{o}}}{\rho_{\mathrm{c}}}\right)^{2}} .
$$

In the case of a shallow oceanic envelope $\left(R_{\mathrm{p}} \simeq R_{\mathrm{c}}\right)$, the height of the oceanic tide is then given by $R_{\mathrm{c}}\left(T_{2}-S_{2}\right)$ at the surface of the core. Using Eqs. (53), (45), we obtain the classical expression of the height of oceanic tide

$$
R_{\mathrm{c}}\left(T_{2}-S_{2}\right)=\frac{\bar{\mu} \zeta_{\mathrm{c}}}{1-\frac{\rho_{\mathrm{o}}}{\rho_{\mathrm{c}}}+\bar{\mu}\left(1-\frac{3}{5} \frac{\rho_{\mathrm{o}}}{\rho_{\mathrm{c}}}\right)}
$$

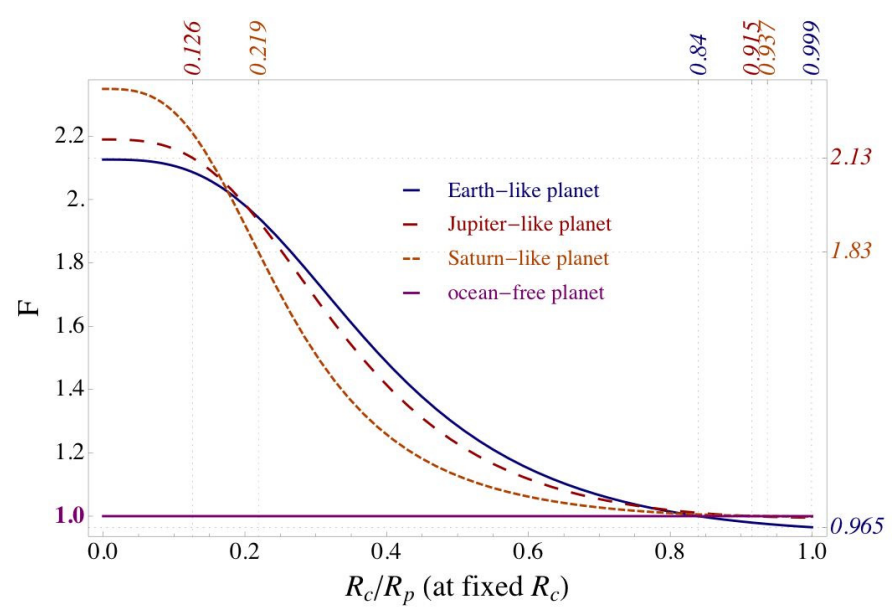

Fig. 5. Factor $F$, accounting for the overloading exerted by the tidally deformed oceanic envelope on the solid core, in terms of the ocean depth through the ratio $R_{\mathrm{c}} / R_{\mathrm{p}}$ for three types of planet. Parameters are given in Tables 4-6: for Earth-, Jupiter-, and Saturn-like planets, we assume respectively that $R_{\mathrm{p}}=\{1,10.97,9.14\}$ (in units of $R_{\mathrm{p}}^{\oplus}$ ), $M_{\mathrm{p}}=$ $\{1,317.83,95.16\}$ (in units of $\left.M_{\mathrm{p}}^{\oplus}\right), R_{\mathrm{c}}=\{0.99,0.126,0.219\} \times R_{\mathrm{p}}$, $M_{\mathrm{c}}=\{0.33,6.41,18.65\}$ (in units of $M_{\mathrm{p}}^{\oplus}$ ), and $\mu=5 \times 10^{10}(\mathrm{~Pa})$ for all cores. Note that this figure is similar to that in Dermott (1979), any differences being caused by the use of different values of the parameters.

Table 4. Earth parameters.

\begin{tabular}{lcl}
\hline \hline Quantity & Value & \multicolumn{1}{c}{ Reference } \\
\hline$M_{\mathrm{p}}^{\oplus}(\mathrm{kg})$ & $5.9736 \times 10^{24}$ & http://nssdc.gsfc.nasa.gov/ \\
$R_{\mathrm{p}}^{\oplus}(\mathrm{m})$ & $6371 \times 10^{3}$ & planetary/factsheet/ \\
$M_{\mathrm{c}}^{\oplus}(\mathrm{kg})$ & $M_{\mathrm{p}}^{\oplus}-1.4 \times 10^{21}$ & earthfact.html \\
$R_{\mathrm{c}}^{\oplus}(\mathrm{m})$ & $R_{\mathrm{p}}^{\oplus}-3682 \times 10^{3}$ & Charette \& Smith $(2010)$ \\
\hline
\end{tabular}

Table 5. Mass and mean radius of Jupiter and Saturn.

\begin{tabular}{lccc}
\hline \hline Quantity & Jupiter & Saturn & Reference \\
\hline$R_{\mathrm{p}}(\mathrm{m})$ & $10.973 \times R_{\mathrm{p}}^{\oplus}$ & $9.140 \times R_{\mathrm{p}}^{\oplus}$ & $\mathrm{http}: / /$ nssdc.gsfc.nasa. \\
$M_{\mathrm{p}}(\mathrm{kg})$ & $317.830 \times M_{\mathrm{p}}^{\oplus}$ & $95.159 \times M_{\mathrm{p}}^{\oplus}$ gov/planetary/factsheet/ \\
\hline
\end{tabular}

The height of the solid tidal displacement is given by $R_{\mathrm{c}} S_{2}$. Using Eqs. (57), (53), (45), we obtain its classical expression

$R_{\mathrm{c}} S_{2}=\frac{\frac{5}{2} \zeta_{\mathrm{c}}\left(1-\frac{\rho_{\mathrm{o}}}{\rho_{\mathrm{c}}}\right)}{1-\frac{\rho_{\mathrm{o}}}{\rho_{\mathrm{c}}}+\bar{\mu}\left(1-\frac{3}{5} \frac{\rho_{\mathrm{o}}}{\rho_{\mathrm{c}}}\right)}$,

which reduces to

$R_{\mathrm{c}} S_{2}=\frac{\frac{5}{2} \zeta_{\mathrm{p}}}{1+\bar{\mu}}$

for an ocean-free planet $\left(\rho_{\mathrm{o}}=0\right)$, corresponding to that given by Lord Kelvin (1863). Thus, recalling Eq. (46), we deduce that for an oceanless planet, $F$ is unity.

Figure 5 displays the value of $F$ for three types of planets (i.e. Earth-, Jupiter-, and Saturn-like planets), with a given core (of fixed size, mass, and shear modulus) and a fluid shell of fixed density but variable depth, such that the size and mass of the whole planet also varies. The variation of $F$ is represented in function of $R_{\mathrm{c}} / R_{\mathrm{p}}$ : the smaller this ratio, the greater the ocean depth.

For the Earth, all parameters are well-known (see Table 4). 
Table 6. Mass and mean radius of Jupiter's and Saturn's cores.

\begin{tabular}{ccc}
\hline \hline$R_{\mathrm{c}}(\mathrm{m})$ & $M_{\mathrm{c}}(\mathrm{kg})$ & Reference \\
\hline Jupiter $0.126 \times R_{\mathrm{p}}$ & $6.41 \times M_{\mathrm{p}}^{\oplus}$ & $\begin{array}{l}\text { http://wWw.oca.eu/guillot/ } \\
\text { jupsat.html (Guillot 1999) }\end{array}$ \\
\hline Saturn $0.219 \times R_{\mathrm{p}}$ & $18.65 \times M_{\mathrm{p}}^{\oplus}$ & Hubbard et al. (2009) \\
\hline
\end{tabular}

The values of the ocean density for the Jupiter- and Saturnlike planets correspond to the ones we may deduce from the well-known values of their global size and mass (see Table 5), and the much more poorly constrained values of the core size and mass (see models A of Table 6). We use the shear modulus taken as reference by Henning et al. (2009) when studying the tidal heating of terrestrial exoplanets, i.e. $\mu=5 \times 10^{10} \mathrm{~Pa}$. These models of planets are used as starting points to compare the influence that the ocean depth has on core deformation for different types of planets. Since we do not try to estimate this deformation for realistic planets, we do not discuss in this section the validity of the values we use for the parameters.

Figure 5 shows that for a planet with a shallow fluid shell (i.e. when $R_{\mathrm{c}} \gtrsim a \times R_{\mathrm{p}}$, where $a=\{0.840,0.915,0.937\}$ for an Earth-, Jupiter-, and Saturn-like planet respectively), $F$ is less than unity, which means that the ocean exerts a loading effect on the solid core that is stronger than the gravitational forces and opposed to it. This is the case for the Earth where the depth of the oceanic envelope does not exceed $1 \%$ of the size of the planet, but giant planets are supposed to have a solid core no bigger than the third of the planet size. According to Fig. 5, F may reach values of up to 2.3 for this kind of planets, meaning that for a planet with a deep fluid envelope, the ocean tide has no loading effect on the core but exerts a gravitational force that amplifies the tidal deformation. We refer the reader to Dermott (1979) for a complete discussion.

\subsection{Modified Love numbers}

From Eq. (56), we deduce the Love number $h_{2}^{F}$ (cf. Eq. (28)), which measures the surface deformation:

$$
h_{2}^{F}=\frac{\frac{5}{2}\left(1-\frac{\rho_{\mathrm{o}}}{\rho_{\mathrm{c}}}\right)\left(1+\frac{3}{2 \alpha}\right)}{1+\bar{\mu}-\frac{\rho_{\mathrm{o}}}{\rho_{\mathrm{c}}}+\frac{3}{2} \frac{\rho_{\mathrm{o}}}{\rho_{\mathrm{c}}}\left(1-\frac{\rho_{\mathrm{o}}}{\rho_{\mathrm{c}}}\right)-\frac{9}{4 \alpha}\left(\frac{R_{\mathrm{c}}}{R_{\mathrm{p}}}\right)^{5}\left(1-\frac{\rho_{\mathrm{o}}}{\rho_{\mathrm{c}}}\right)^{2}} .
$$

We give here the expression of the second-order Love number (20) associated with the solid core. First of all, we recall its value for an ocean-free planet. According to Eq. (29) with $r=R_{\mathrm{c}}$, we get

$k_{2}=\frac{3}{5} h_{2}=\frac{3}{2} \frac{1}{1+\bar{\mu}}$.

In the presence of an ocean on top of the solid core, we may also introduce the modified Love number

$k_{2}^{F} \stackrel{\text { def }}{=} \frac{\Phi^{\prime}\left(R_{\mathrm{c}}\right)}{U\left(R_{\mathrm{c}}\right)}=\frac{V^{\prime}\left(R_{\mathrm{c}}\right)-U\left(R_{\mathrm{c}}\right)}{U\left(R_{\mathrm{c}}\right)}=\frac{V^{\prime}\left(R_{\mathrm{c}}\right)}{U\left(R_{\mathrm{c}}\right)}-1$,

where $V^{\prime}\left(R_{\mathrm{c}}\right)$ and $U\left(R_{\mathrm{c}}\right)$ are obtained from Eqs. (31b) and (21), respectively, with $r=R_{\mathrm{c}}$. Thus, expressing $\zeta_{\mathrm{c}} / R_{\mathrm{c}}$ as a function of the modified second-order Love number $h_{2}^{F}$ according to Eq. (28), and using Eq. (45), we obtain the expression of $k_{2}^{F}$ in terms of $h_{2}^{F}$

$k_{2}^{F}=\left(1+\frac{2}{5} \frac{\bar{\mu}}{1-\frac{\rho_{0}}{\rho_{\mathrm{c}}}}\right) h_{2}^{F}-1$.
In this section, we have studied the impact of the presence of a fluid envelope on the determination of the deformation imposed on an elastic core under tidal forcing. In the following, we consider that the solid core also has viscous properties such that its response to the tidal force exerted by the perturber is no more immediate, thus inducing dissipation. The next section addresses the quantification of this conversion of energy, which drives the dynamical evolution of the whole system.

\section{Anelastic tidal dissipation: analytical results}

Assuming that the anelasticity is linear, the correspondence principle established by Biot (1954) allows us to extend the formulation of the adiabatic elastic problem to the resolution of the equivalent dissipative anelastic problem. For initial conditions taken as zero and similar geometries, the Laplace and Fourier transforms of the anelastic equations and boundary conditions are identical to the elastic equations, if the rheological parameters and radial functions are defined as complex numbers. We then denote as

$\tilde{\tilde{\boldsymbol{\sigma}}} \equiv \overline{\bar{\sigma}}_{1}+\mathrm{i} \overline{\bar{\sigma}}_{2}$

the complex stress tensor, and as

$\widetilde{\widetilde{\epsilon}} \equiv \overline{\bar{\epsilon}}_{1}+\mathrm{i} \overline{\bar{\epsilon}}_{2}$

the complex strain tensor.

The perturbative strain is cyclic, with tidal pulsations $\sigma_{2, m, p, q}$. For sake of clarity, we use the generic notation $\omega \equiv \sigma_{2, m, p, q}$, recalling that there is a large range of tidal frequencies for each term of the expansion of the tidal potential. The stress and strain tensors take the form

$\tilde{\tilde{\boldsymbol{\sigma}}}(\omega)=\left(\overline{\overline{\boldsymbol{\sigma}}}_{1}+\mathrm{i} \overline{\overline{\boldsymbol{\sigma}}}_{2}\right) \mathrm{e}^{\mathrm{i} \omega t}$,

$\widetilde{\tilde{\boldsymbol{\epsilon}}}(\omega)=\widetilde{\boldsymbol{\epsilon}}_{0} \mathrm{e}^{\mathrm{i} \omega t}$.

The complex rigidity

$\tilde{\mu}(\omega) \equiv \mu_{1}(\omega)+\mathrm{i} \mu_{2}(\omega)$

where $\mu_{1}$ represents the energy storage and $\mu_{2}$ the energy loss of the system, is defined by

$\tilde{\mu}(\omega) \equiv \frac{\tilde{\tilde{\boldsymbol{\sigma}}}(\omega)}{\tilde{\boldsymbol{\epsilon}}(\omega)}$

We may also define the complex effective rigidity

$\hat{\mu}(\omega) \equiv \bar{\mu}_{1}(\omega)+\mathrm{i} \bar{\mu}_{2}(\omega)$

by

$\hat{\mu}(\omega)=\gamma \tilde{\mu}(\omega)$,

where (see Eq. (27))

$\gamma=\frac{\hat{\mu}}{\tilde{\mu}} \equiv \frac{\bar{\mu}}{\mu}=\frac{19}{2 \rho_{\mathrm{c}} g_{\mathrm{c}} R_{\mathrm{c}}}$. 


\subsection{Case of a fully-solid planet}

The complex Love number $\tilde{k}_{2}$ may be expressed in terms of the complex effective rigidity $\hat{\mu}$, by:

$$
\begin{aligned}
\tilde{k}_{2}(\omega) & =\frac{3}{2} \frac{1}{1+\hat{\mu}(\omega)}, \\
& =\frac{3}{2} \frac{1}{1+\gamma\left[\mu_{1}(\omega)+\mathrm{i} \mu_{2}(\omega)\right]},
\end{aligned}
$$

in the case of a completely solid planet.

The real part of $\tilde{k}_{2}$ characterizes the purely elastic deformation, since $-\operatorname{Im}\left(\tilde{k}_{2}\right)$ gives the phase lag due to the viscosity. Therefore, we define the factor of tidal dissipation $Q$, which represents the dissipation rate due to viscous friction, by

$Q^{-1}(\omega)=-\frac{\operatorname{Im} \tilde{k}_{2}(\omega)}{\left|\tilde{k}_{2}(\omega)\right|}$

From Eq. (72), we then deduce that

$Q(\omega)=\sqrt{1+\left[\frac{1}{\bar{\mu}_{2}(\omega)}+\frac{\bar{\mu}_{1}(\omega)}{\bar{\mu}_{2}(\omega)}\right]^{2}}$.

\subsection{Case of a two-layer planet}

We introduce the quantities

$A=\left(1-\frac{\rho_{\mathrm{o}}}{\rho_{\mathrm{c}}}\right)\left(1+\frac{3}{2 \alpha}\right)$, and

$B=1-\frac{\rho_{\mathrm{o}}}{\rho_{\mathrm{c}}}+\frac{3}{2} \frac{\rho_{\mathrm{o}}}{\rho_{\mathrm{c}}}\left(1-\frac{\rho_{\mathrm{o}}}{\rho_{\mathrm{c}}}\right)-\frac{9}{4 \alpha}\left(\frac{R_{\mathrm{c}}}{R_{\mathrm{p}}}\right)^{5}\left(1-\frac{\rho_{\mathrm{o}}}{\rho_{\mathrm{c}}}\right)^{2}$,

into Eq. (56) for $F$

$F=\frac{A(1+\bar{\mu})}{B+\bar{\mu}}$

and we define its complex equivalent

$\widetilde{F}=\frac{A(1+\hat{\mu})}{B+\hat{\mu}}$.

The determination of how the presence of an oceanic envelope can modify the tidal dissipation consists in the determination of $\tilde{k}_{2}^{F}$, which is defined as the complex Love number $\tilde{k}_{2}$ in presence of the fluid envelope. According to the correspondence principle, this number is given by the complex Fourier transform of Eq. (63), i.e.

$\tilde{k}_{2}^{F}(\omega)=\left(1+\frac{2}{5} \frac{\hat{\mu}(\omega)}{1-\frac{\rho_{\mathrm{o}}}{\rho_{\mathrm{c}}}}\right) \tilde{h}_{2}^{F}(\omega)-1$.

From (63) we get then

$$
\begin{aligned}
\tilde{k}_{2}^{F}(\omega)= & \frac{1}{\left(B+\bar{\mu}_{1}\right)^{2}+\bar{\mu}_{2}^{2}} \\
& \times\left\{\left[\left(B+\bar{\mu}_{1}\right)\left(C+\frac{3}{2 \alpha} \bar{\mu}_{1}\right)+\frac{3}{2 \alpha} \bar{\mu}_{2}^{2}\right]-\mathrm{i} A D \bar{\mu}_{2}\right\},
\end{aligned}
$$

where we have used the dimensionless quantities $\alpha, A$, and $B$ previously defined (see respectively Eqs. (54) and (75)), where $C$ and $D$ are given by

$C=\frac{3}{2}\left(1-\frac{\rho_{\mathrm{o}}}{\rho_{\mathrm{c}}}\right)\left(1-\frac{\rho_{\mathrm{o}}}{\rho_{\mathrm{c}}}+\frac{5}{2 \alpha}\right)+\frac{9}{4 \alpha}\left(\frac{R_{\mathrm{c}}}{R_{\mathrm{p}}}\right)^{5}\left(1-\frac{\rho_{\mathrm{o}}}{\rho_{\mathrm{c}}}\right)^{2}$,

$D=\frac{3}{2}\left(1-\frac{\rho_{\mathrm{o}}}{\rho_{\mathrm{c}}}\right)\left[1+\frac{3}{2 \alpha}\left(\frac{R_{\mathrm{c}}}{R_{\mathrm{p}}}\right)^{5}\right]$.
Finally, the dissipation factor $\hat{Q}$, defined here by

$\hat{Q}^{-1}(\omega)=-\frac{\operatorname{Im} \tilde{k}_{2}^{F}(\omega)}{\left|\tilde{k}_{2}^{F}(\omega)\right|}$,

is of the form

$\hat{Q}(\omega)=\sqrt{1+\frac{9}{4 \alpha^{2} A^{2} D^{2}}\left[1+\frac{\left(B+\bar{\mu}_{1}(\omega)\right)\left(\frac{2 \alpha C}{3}+\bar{\mu}_{1}(\omega)\right)}{\bar{\mu}_{2}(\omega)}\right]^{2}}$.

Thanks to the correspondence principle, one is able to derive this general expression of the tidal dissipation, which is valid for any rheology. The dependence on the tidal frequency $\omega \equiv \sigma_{2, m, p, q}$ of the derived formulae is clearly evident, as shown, for example, by Remus et al. (2012), and Ogilvie \& Lin (2004-2007) for fluid layers.

\subsection{Implementation of an anelastic model}

Since the factor $Q$ depends on the real and imaginary components $\bar{\mu}_{1}$ and $\bar{\mu}_{2}$ of the complex effective shear modulus $\hat{\mu}$, we need to define the rheology of the studied body to express it in terms of the constitutive parameters of the material.

The anelasticity of a material is evaluated by a quality factor $Q_{\mathrm{a}}$ defined by

$Q_{\mathrm{a}}(\omega)=\frac{\mu_{1}(\omega)}{\mu_{2}(\omega)}$.

We can express the solid tidal dissipation, given by $Q$ (Eq. (74)), for a fully-solid planet and $\hat{Q}$ (Eq. (82)) in the case of a two-layer planet, in terms of $Q_{\mathrm{a}}$, as

$Q\left(\omega \equiv \sigma_{2, m, p, q}\right)=\sqrt{1+\left[\frac{1}{\bar{\mu}_{2}(\omega)}+Q_{\mathrm{a}}(\omega)\right]^{2}}$,

and

$$
\begin{aligned}
& \hat{Q}\left(\omega \equiv \sigma_{2, m, p, q}\right) \\
& =\sqrt{1+\frac{9}{4 \alpha^{2} A^{2} D^{2}}\left[1+\left(\frac{B}{\bar{\mu}_{2}(\omega)}+Q_{\mathrm{a}}(\omega)\right)\left(\frac{2}{3} \frac{\alpha C}{\bar{\mu}_{2}(\omega)}+Q_{\mathrm{a}}(\omega)\right)\right]^{2}} .
\end{aligned}
$$

All previous results are independent of the viscoelastic rheological model. We now apply these general expressions to a specific rheology that depends on the physical properties of the material. Given our lack of knowledge of the internal structure of giant planets, we implement the simplest model, namely the Maxwell model, which has the advantage of involving only two parameters and is thus easy to use (Tobie 2003; Tobie et al. 2005). A critical overview of the four main rheological models was done by Henning et al. (2009), thus we refer the reader to the three aforementioned papers for a detailed comparison.

\subsection{The Maxwell model}

This model considers a viscoelastic material as a spring-dashpot series. The instantaneous elastic response is characterized by a shear modulus $G$, and the viscous yielding is represented by a viscous scalar modulus $\eta$ (see Fig. 6). Note that the shear moduli $G$ and $\mu$ (introduced in Sect. 2.4) designate the same quantity. We 


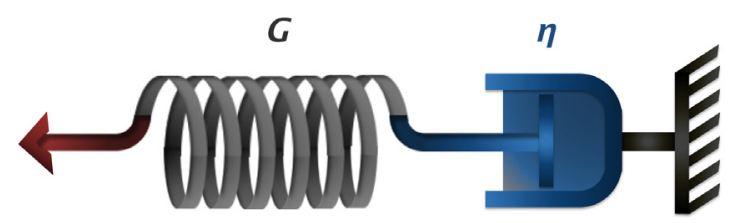

Fig. 6. Representation of the Maxwell model and its corresponding notations.

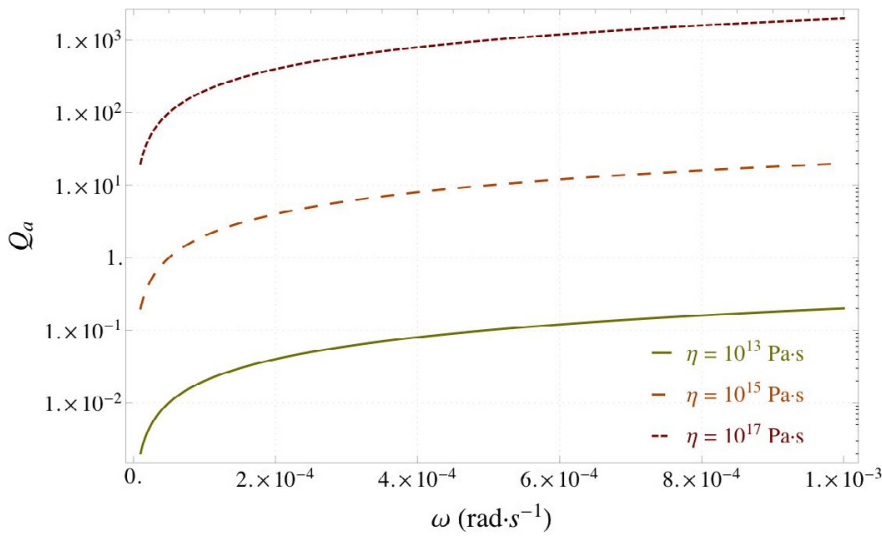

Fig. 7. Anelastic quality factor $Q_{\mathrm{a}}$ of the Maxwell model in function of the tidal pulsation $\omega$ for different values of the viscosity $\eta$. $\mathrm{G}$ is taken equal to $5 \times 10^{10} \mathrm{~Pa}$ (see Henning et al. 2009). $Q_{\mathrm{a}}$ is represented on a logarithmic scale.

change here the notation to avoid any confusion with the complex shear modulus $\tilde{\mu}$ used to study the anelastic tidal dissipation, whose real and imaginary parts involve both $G$ and $\eta$.

The constitutive equation is given by Henning et al. (2009)

$G \tilde{\tilde{\sigma}}(\omega)+\eta \dot{\tilde{\sigma}}(\omega)=G \eta \dot{\tilde{\boldsymbol{\epsilon}}}(\omega)$,

where the time derivative of a given quantity is denoted by a dot. Recalling Eq. (68), this equation becomes

$G \tilde{\tilde{\boldsymbol{\epsilon}}}(\omega) \tilde{\mu}(\omega)+\eta \frac{\mathrm{d}}{\mathrm{d} t}[\tilde{\mu}(\omega) \tilde{\tilde{\boldsymbol{\epsilon}}}(\omega)]=G \eta \dot{\tilde{\boldsymbol{\epsilon}}}(\omega)$.

Therefore the real part $\mu_{1}$ and the imaginary part $\mu_{2}$ of the complex shear modulus $\tilde{\mu}$ are given by

$\mu_{1}(\omega)=\frac{\eta^{2} G \omega^{2}}{G^{2}+\eta^{2} \omega^{2}}$

$\mu_{2}(\omega)=\frac{\eta G^{2} \omega}{G^{2}+\eta^{2} \omega^{2}}$.

The anelastic quality factor $Q_{\mathrm{a}}$ is then given by

$Q_{\mathrm{a}}(\omega)=\frac{\mu_{1}(\omega)}{\mu_{2}(\omega)}=\frac{\eta \omega}{G} \equiv \omega \tau_{\mathrm{M}}$,

where $\tau_{\mathrm{M}}=\eta / G$ is the characteristic time of relaxation of the Maxwell model. As confirmed by Fig. 7, Eq. (89) shows that $Q_{\mathrm{a}}$ increases linearly with the frequency of the cyclic tidal strain: the shorter the oscillation period, the lower the dissipation due to the intrinsic viscoelastic properties of the material. Moreover, the anelastic quality factor is proportional to $\tau_{\mathrm{M}}=\eta / G$, such that it dissipates more if it is more rigid and less viscous.

Thus, we may express $\mu_{2}$ (Eq. (88b)) in terms of the anelastic quality factor $Q_{\mathrm{a}}$ (Eq. (89))

$\mu_{2}(\omega)=\frac{G}{\left(\omega \tau_{M}\right)^{-1}+\omega \tau_{M}}$.

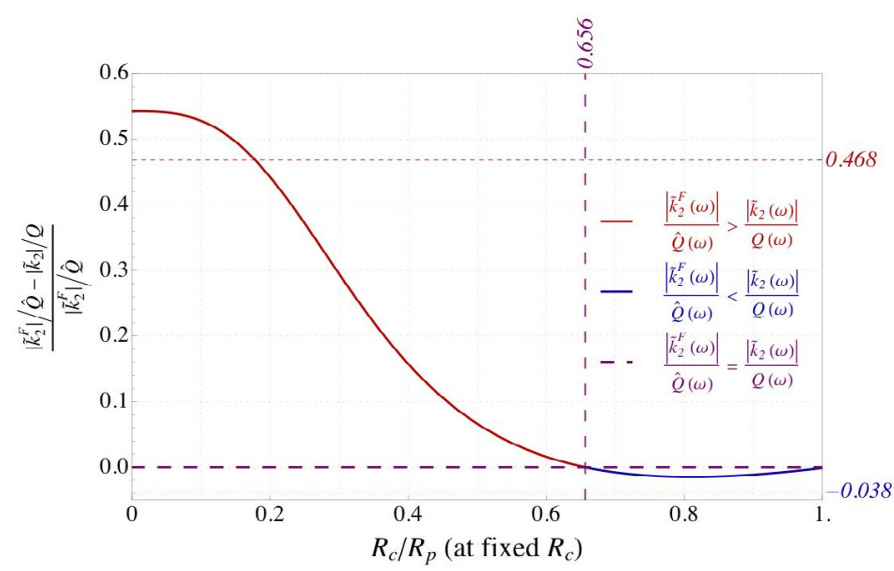

Fig. 8. Relative difference between $\left|\tilde{k}_{2}^{F}\right| / \hat{Q}$ and $\left|\tilde{k}_{2}\right| / Q$. We may distinguish two regimes: for $0<R_{\mathrm{c}}<0.661 R_{\mathrm{p}}$ the fluid envelope increases the tidal dissipation; above this value, tidal dissipation is lower than it would have been without a fluid shell. Parameters are given in Tables 5-7 for a Saturn-like planet perturbed at the tidal frequency of Enceladus $\left(\omega=2.25 \times 10^{-4} \mathrm{rad} \mathrm{s}^{-1}\right): R_{\mathrm{p}}=9.14 R_{\mathrm{p}}^{\oplus}, M_{\mathrm{p}}=95.16 M_{\mathrm{p}}^{\oplus}$, $R_{\mathrm{c}}=0.219 R_{\mathrm{p}}^{\oplus}, M_{\mathrm{c}}=18.65 M_{\mathrm{p}}^{\oplus}, G=5 \times 10^{10}(\mathrm{~Pa})$, and $\eta=10^{15}(\mathrm{~Pa} \mathrm{~s})$.

Table 7. Tidal frequencies considered in numerical applications.

\begin{tabular}{lcc}
\hline \hline Planet & Jupiter & Saturn \\
\hline Satellite & Io & Enceladus \\
$\omega\left(\mathrm{rad} \mathrm{s}^{-1}\right)$ & $2.79 \times 10^{-4}$ & $2.25 \times 10^{-4}$ \\
Reference & Ioannou et Lindzen (1993) & Lainey et al. (2012) \\
\hline
\end{tabular}

In the case of a fully-solid body, we get, from Eqs. (83) and (90), the imaginary part of the complex Love number $\tilde{k}_{2}(72)$

$\operatorname{Im}\left[\tilde{k}_{2}(\omega)\right]=-\frac{3 \gamma}{2} \frac{G \omega \tau_{\mathrm{M}}}{1+\left(\omega \tau_{\mathrm{M}}\right)^{2}(1+\gamma G)^{2}}$.

Therefore, the dissipation factor $Q$ defined by Eq. (74) is of the form

$Q\left(\omega \equiv \sigma_{2, m, p, q}\right)=\sqrt{1+\left\{\frac{1}{G \gamma}\left[\left(\omega \tau_{\mathrm{M}}\right)^{-1}+\omega \tau_{\mathrm{M}}\right]+\omega \tau_{\mathrm{M}}\right\}^{2}}$.

In the more general case of a two-layer body, the imaginary part of the complex Love number $\tilde{k}_{2}^{F}$ given by Eq. (79), takes a different form than Eq. (91) because of the presence of the fluid envelope, as does the two-layer dissipation factor $\hat{Q}$ in Eq. (82) with respect to its oceanless form in Eq. (92). To obtain them, one needs to replace the shear modulus $\tilde{\mu}$ and the anelastic quality factor $Q_{\mathrm{a}}$ by their expression in the case of the Maxwell model (Eqs. (88) and (89), respectively).

Figure 8 compares the dissipation of the solid core with and without a fluid envelope of variable depth for a Saturn-like planet, using the parameters given by Tables 5-7: as expected, the difference between the two dissipations decreases with the size of the fluid envelope to about $0.34 \times R_{\mathrm{p}}$; but for a thiner fluid shell, the dissipation get lower than it would be without it.

\section{Anelastic tidal dissipation: role of the structural and rheological parameters}

Owing to our choice of the Maxwell model to represent the rheology of the solid parts of the planet, the dissipation quality factor $\hat{Q}$ depends on both the tidal frequency $\omega$ and four structural 
and rheological parameters: the relative size of the core $\left(R_{\mathrm{c}} / R_{\mathrm{p}}\right)$, the relative density of the envelope with respect to the core $\left(\rho_{\mathrm{o}} / \rho_{\mathrm{c}}\right)$, the shear modulus $(G)$, and the viscosity of the core $(\eta)$. Our present knowledge of extrasolar giant planets, in addition to the planets of our solar system like Jupiter or Saturn, is affected by some uncertainties in the values of these parameters, such that their ranges are poorly constrained. Moreover, even if the presence of a core in Jupiter has not yet been confirmed (see Guillot 1999-2005), seismological data may provide more constraints on giant-planet internal structure (see Gaulme et al. 2011). Nevertheless, we explore the resulting tidal dissipation in these bodies around values of the structural and rheological parameters taken as reference and corresponding to those of the literature.

\subsection{Baseline structural and rheological parameters}

As reference models, we chose Jupiter and Saturn, although their core parameters remain uncertain. The values of the global sizes and masses of these planets are those of Table 5 .

\subsubsection{Size and mass of the core}

Two main types of models are presently available for Jupiter's interior. The NHKFRB group ${ }^{1}$ uses a three-layer model with a thin radiative zone, close to previous models by Saumon \& Guillot (2004), whereas the MHVTB group ${ }^{2}$ proposes a new type of Jupiter model that has only two layers (see Militzer et al. 2008). As explained in Militzer \& Hubbard (2009), the crucial difference between the two lies in their treatments of the molecular-to-metallic transition in dense fluid hydrogen, leading to very different conclusions. The first group predicts a core that is smaller than $10 M_{\mathrm{p}}^{\oplus}$ (Saumon \& Guillot 2004), while the second one obtains a larger core of $14-18 M_{\mathrm{p}}^{\oplus}$ (Militzer et al. 2008). Among all these models of Jupiter's interior, we choose as reference the adiabatic model with plasma phase transition (PPT) ${ }^{3}$ of Guillot (1999), which is of the first type. It predicts a core of radius $R_{\mathrm{c}}=0.126 \times R_{\mathrm{p}}$ and mass $M_{\mathrm{c}}=6.41 \times M_{\mathrm{p}}^{\oplus}$. Only the mass of the core of this reference model is used in what follows. The core radius just serves as a first approximation, as a starting point in our study, since we present our results for several core sizes.

There are also different models of Saturn's interior. According to the model of Guillot with PPT $^{3}$, Saturn's core may have a mass of $M_{\mathrm{c}}=6.55 \times M_{\mathrm{p}}^{\oplus}$ and a size of $R_{\mathrm{c}}=0.174 \times R_{\mathrm{p}}$. Hubbard et al. (2009) infered, from Cassini-Huygens data, that Saturn has a larger core in the range $M_{\mathrm{c}}=15-20 \times M_{\mathrm{p}}^{\oplus}$ and a corresponding radius of more than $20 \%$ of the planet size. We adopt this latter as reference model of Saturn, with $M_{\mathrm{c}}=18.65 \times M_{\mathrm{p}}^{\oplus}$ and $R_{\mathrm{c}}=0.219 \times R_{\mathrm{p}}$.

All these models assume that core accretion is the standard process for the formation of giant planets, the corresponding parameters being listed in Table 6 .

\subsubsection{Rheological parameters of the core}

The main uncertainties concern the viscoelastic properties of the core, namely its shear modulus $G$ and its viscosity $\eta$. At high

\footnotetext{
1 Nettelmann, Holst, Kietzmann, French, Redmer and Blaschke (Nettelmann et al. 2008).

2 Militzer, Hubbard, Vorberger, Tamblyn, and Bonev (Militzer et al. 2008).

3 The model is available at http://www.oca.eu/guillot/jupsat. html. It is constructed with CEPAM, Code d'Évolution Planétaire Adaptatif et Modulaire (Guillot \& Morel 1995).
}

pressure and temperature, theoretical models and experiments show that $G$ and $\eta$ values depend on temperature and pressure. however, no experiments are available at the very-high pressures and temperatures we may expect in Jupiter's and Saturn's cores (Guillot 2005). Nevertheless, geophysical and experimental data allow to constrain the rheology of the icy satellites of Jupiter, since their ranges of pressure and temperature are similar to those of the outer mantle of the Earth (Tobie 2003). Then, keeping in mind that these values may differ by several orders of magnitude in our case, we adopt reference values based on these data, assuming that Jupiter's and Saturn's cores are made of ice and rock. We then explore, in Sect. 5.2, the variation of the tidal dissipation for a large range of values of the rheological parameters around those taken as reference.

We thus assume that the shear modulus $G$ is in the range $\left[G_{\text {ice }}=4 \times 10^{9}(\mathrm{~Pa}), G_{\text {silicate }}=10^{11}(\mathrm{~Pa})\right]($ Henning et al. 2009).

The viscosity $\eta$ has values in the range $\left[\eta_{\text {ice }}=10^{14} \mathrm{Pas}\right.$, $\eta_{\text {silicate }}=10^{21} \mathrm{~Pa}$ s] for the icy satellites of Jupiter at high pressure (Tobie 2003). We expand this range, by reducing its lower boundary by two orders of magnitude. This is in line with the discussion of Karato (2011), which seems to indicate that, at the very high pressures, viscosity in the deep interior of super-Earths may decrease by two or three orders of magnitude. We refer the reader to Karato (2011) for an overview of all plausible mechanisms that may change the viscous-pressure relationship at veryhigh pressures.

\subsection{Dependence of tidal dissipation on rheology}

Since tidal dissipation causes exchange of angular momentum in the system, it may be quantified by monitoring carefuly the orbital motion of the system. Using astrometric data covering more than a century, Lainey et al. (2009-2012) succeeded in determining from observations the tidal dissipation in Jupiter and Saturn: namely, $Q_{\text {Jupiter }}=(3.56 \pm 0.56) \times 10^{4}$ determined by Lainey et al. (2009), and $Q_{\text {Saturn }}=(1.682 \pm 0.540) \times 10^{3}$ determined by Lainey et al. (2012) and requireded by the formation scenario of Charnoz et al. (2011). However, with this method, the different contributions to the global tidal dissipation, from each layer constituting the planet, are combined together. Equations of the dynamical evolution (Eqs. (99) to (103)) link the observed evolution rates of the rotational and orbital parameters to both the observed tidal dissipation and system characteristics. Since all these rates are proportional to $R_{\mathrm{p}}^{5}$, where $R_{\mathrm{p}}$ is the planet radius, we introduce the associated dissipation factor ${ }^{4}$

$\hat{Q}_{\mathrm{eff}}=\left(\frac{R_{\mathrm{p}}}{R_{\mathrm{c}}}\right)^{5} \times \frac{\left|\tilde{k}_{2}^{F}\left(R_{\mathrm{p}}\right)\right|}{\left|\tilde{k}_{2}^{F}\left(R_{\mathrm{c}}\right)\right|} \times \hat{Q}$,

where $\left|\tilde{k}_{2}^{F}\left(R_{\mathrm{c}}\right)\right|$ can be deduced from Eq. (81), and $\left|\tilde{k}_{2}^{F}\left(R_{\mathrm{p}}\right)\right|$ designates the modulus of the second order Love number of the planet's surface that is obtained from Eqs. (52) and (21)

$\tilde{k}_{2}^{F}\left(R_{\mathrm{p}}\right)=\frac{V^{\prime}\left(R_{\mathrm{p}}\right)}{U\left(R_{\mathrm{p}}\right)}-1=\frac{\frac{\rho_{\mathrm{o}}}{\rho_{\mathrm{c}}}-\left(\frac{R_{\mathrm{c}}}{R_{\mathrm{p}}}\right)^{3}\left(1-\frac{\rho_{\mathrm{o}}}{\rho_{\mathrm{c}}}\right)}{\frac{2}{5} \frac{\rho_{\mathrm{o}}}{\rho_{\mathrm{c}}} \alpha-\frac{3}{5}\left(\frac{R_{\mathrm{c}}}{R_{\mathrm{p}}}\right)^{5}\left(1-\frac{\rho_{\mathrm{o}}}{\rho_{\mathrm{c}}}\right)^{2} \frac{\rho_{\mathrm{o}}}{\rho_{\mathrm{c}}}\left(\alpha+\frac{3}{2}\right) \frac{1}{H}}$,

where $H$ accounts for the quantity

$H=1+\tilde{\mu}-\frac{\rho_{\mathrm{o}}}{\rho_{\mathrm{c}}}+\frac{3}{2} \frac{\rho_{\mathrm{o}}}{\rho_{\mathrm{c}}}\left(1-\frac{\rho_{\mathrm{o}}}{\rho_{\mathrm{c}}}\right)+\frac{3}{2}\left(\frac{R_{\mathrm{c}}}{R_{\mathrm{p}}}\right)^{5}\left(1-\frac{\rho_{\mathrm{o}}}{\rho_{\mathrm{c}}}\right)^{2}$.

4 This can also be demonstrated by calculating the perturbation of the gravific potential at the surface of the planet, adapting the theory of Zahn $(1966 a, b)$ to take into account the boundary conditions at the core surface. 

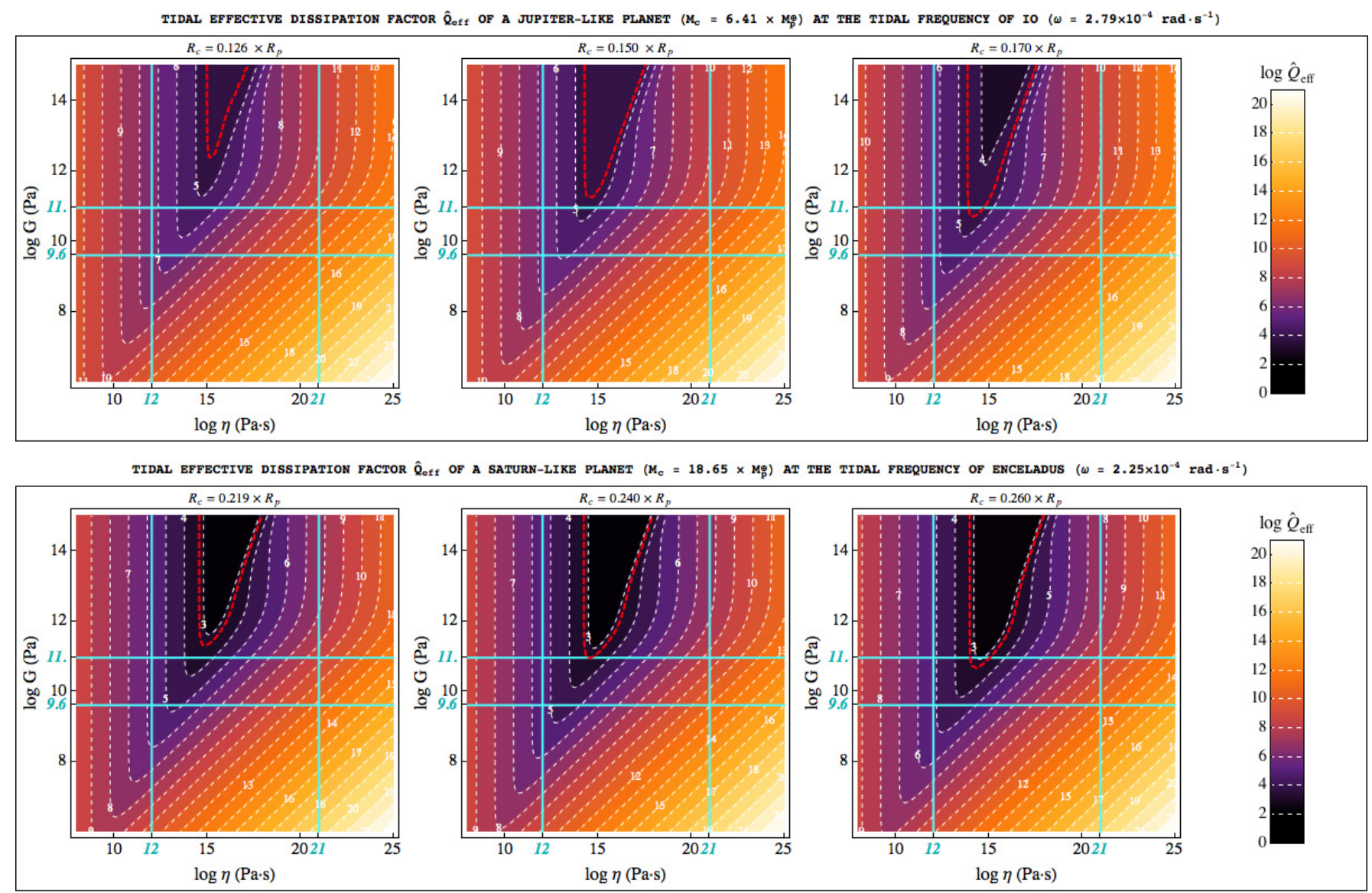

Fig. 9. Dissipation quality factor $\hat{Q}_{\text {eff }}$ of the Maxwell model as a function of the viscoelastic parameters $G$ and $\eta$. Top: for a Jupiter-like two-layer planet tidally perturbed at the Io's frequency $\omega \simeq 2.79 \times 10^{-4} \mathrm{rad} \mathrm{s}^{-1}$. Bottom: for a Saturn-like two-layer planet tidally perturbed at the Enceladus' frequency $\omega \simeq 2.25 \times 10^{-4} \mathrm{rad} \mathrm{s}^{-1}$. The red dashed line corresponds to the value of $\hat{Q}_{\text {eff }}=\left\{(3.56 \pm 0.56) \times 10^{4},(1.682 \pm 0.540) \times 10^{3}\right\}$ (for Jupiter and Saturn, respectively) determined by Lainey et al. (2009-2012). The blue lines corresponds to the lower and upper limits to the reference values taken by the viscoelastic parameters $G$ and $\eta$ for an unknown mixture of ice and silicates. We assume the values of $R_{\mathrm{p}}=\{10.97,9.14\}$ (in units of $\left.R_{\mathrm{p}}^{\oplus}\right), M_{\mathrm{p}}=\{317.8,95.16\}$ (in units of $M_{\mathrm{p}}^{\oplus}$ ), and $M_{\mathrm{c}}=\{6.41,18.65\} \times M_{\mathrm{p}}^{\oplus}$ given in Tables 5, 6.

Since we have weak constraints on the viscoelastic parameters of giant-planet cores (Guillot 2005), we thus have to explore a large range of values. Figure 9 shows the tidal dissipation factor $\hat{Q}_{\text {eff }}$ around the reference values presented in Sect. 5.1, where we expand the range by up to about $\pm 2-4$ orders of magnitude for $G$ and $\eta$. In the middle region (inside the blue rectangle on Fig. 9), where $\eta$ and $G$ correspond to the reference values, the dissipation factor $\hat{Q}_{\text {eff }}$ of Saturn (resp. Jupiter) may reach values of the order of $10^{3}$ (resp. $10^{4}$ ), and in the whole field it varies up to a value of $10^{20}$.

From Fig. 9, we deduce that the tidal dissipation of the core may reach the values observed for Jupiter (Lainey et al. 2009) and Saturn (Lainey et al. 2012) assuming that Jupiter's core (resp. Saturn's core) has a radius 34.92\% (resp. 18.72\%) larger than this of Guillot 1999 (resp. Hubbard 2009).

Therefore, we can evaluate the real part of the second order Love numbers $\tilde{k}_{2}^{F}\left(R_{\mathrm{c}}\right)$ and $\tilde{k}_{2}^{F}\left(R_{\mathrm{p}}\right)$, accounting respectively for the deformation of the core's and planet's surface, for parameters whose values are compatible with the tidal dissipation observations (Lainey et al. 2009-2012). For Jupiter and Saturn, in this order, assuming that $R_{\mathrm{c}}=\{0.170,0.260\} \times R_{\mathrm{p}}, G=$ $\{4.85,4.45\} \times 10^{10} \mathrm{~Pa}$ and $\eta=\{1.26,1.78\} \times 10^{14} \mathrm{~Pa} \mathrm{~s}$, we obtain that $\operatorname{Re}\left[\tilde{k}_{2}^{F}\left(R_{\mathrm{c}}\right)\right]=\{3.21,3.31\}$ and $\operatorname{Re}\left[\tilde{k}_{2}^{F}\left(R_{\mathrm{p}}\right)\right]=\{1.37,0.24\}$. These estimations at the planet's surface can be compared to the value of Gavrilov \& Zharkov (1977) of $k_{2}=0.379$ for Jupiter and $k_{2}=0.341$ for Saturn obtained for stratified models. As discussed in the aforementioned paper, the differences between both evaluations are linked to the degree of stratification: the more the planet interior is stratified, the smaller the second order Love number (we recall that the second order Love number of a homogeneous fluid planet is $3 / 2$ ).

\subsection{Dependence of tidal dissipation on both the size of the core and the tidal frequency}

In Fig. 10, the values of the viscoelastic parameters $G$ and $\eta$, and the core size $R_{\mathrm{c}}$ are based on the results in Fig. 9: they were chosen to ensure that the tidal dissipation factor $\hat{Q}$ reaches the observed values of Lainey et al. (2009-2012) on the condition that the rheological parameters are in the more realistic domain defined by the lowest and highest values of the ice and rock viscoelasticities taken as reference. Taking into account the global dissipation values obtained by Lainey et al. (2009-2012) for Jupiter and Saturn, we can infer some constraints on both the viscoelastic parameters and the size of the core (looking at the red dashed line in Fig. 9). We thus assume that they take values that allow for such a dissipation:

- we first chose a core slightly larger than that assumed until now: $R_{\mathrm{c}}=0.170 \times R_{\mathrm{p}}$ for Jupiter, and $R_{\mathrm{c}}=0.260 \times R_{\mathrm{p}}$ for Saturn; 

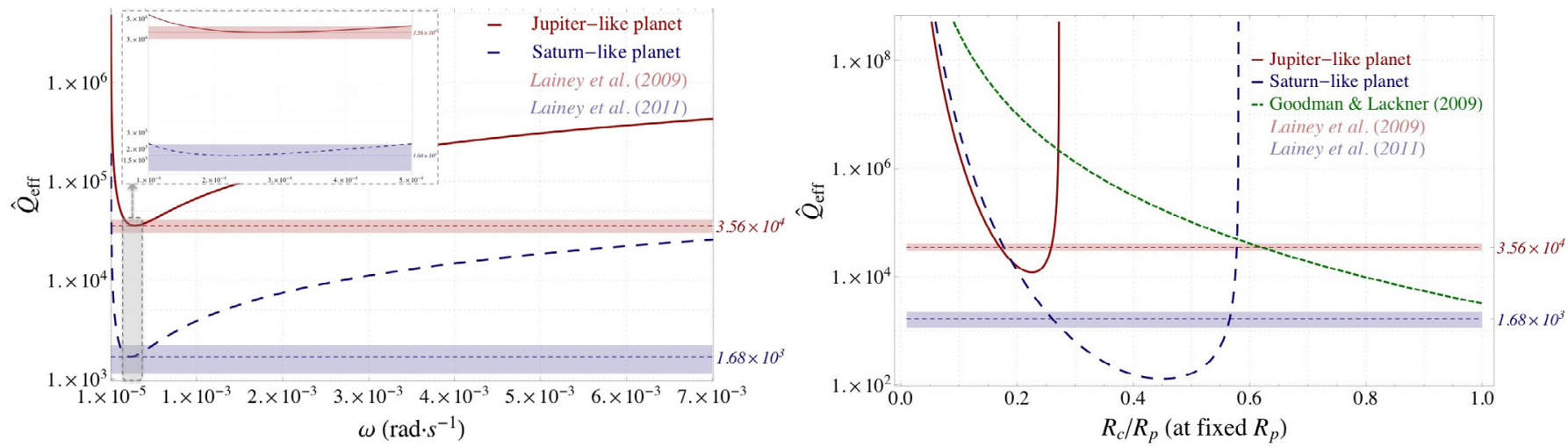

Fig. 10. Dissipation quality factor $\hat{Q}_{\text {eff }}$ normalized to the size of the planet for Jupiter-like and Saturn-like giant planets. Note that all curves are represented on a logarithmic scale. Left: dependence on the perturbative strain pulsation $\omega$, with $R_{\mathrm{c}}=\{0.17,0.26\} \times R_{\mathrm{p}}$. Right: dependence on the size of the core, with $\omega \simeq 2.25 \times 10^{-4} \mathrm{rad} \mathrm{s}^{-1}$ (tidal frequency of Enceladus) for the blue curve associated with a Saturn-like planet, and $\omega \simeq 2.79 \times 10^{-4} \mathrm{rad} \mathrm{s}^{-1}$ (tidal frequency of Io) for the red curve associated with a Jupiter-like planet. The green curve corresponds to the prescription of Goodman \& Lackner (2009) (see plain text for details). The red and blue dashed lines correspond to the value of $\hat{Q}_{\text {eff }}=\left\{(3.56 \pm 0.56) \times 10^{4},(1.682 \pm 0.540) \times 10^{3}\right\}$ (for Jupiter and Saturn, respectively) determined by Lainey et al. (2009-2012). Their zone of uncertainty is also represented by the corresponding color. We assume the values of $R_{\mathrm{p}}=\{10.97,9.14\}$ (in units of $R_{\mathrm{p}}^{\oplus}$ ), $M_{\mathrm{p}}=\{317.8,95.16\}$ (in units of $M_{\mathrm{p}}^{\oplus}$ ), and $M_{\mathrm{c}}=\{6.41,18.65\} \times M_{\mathrm{p}}^{\oplus}$ given in Tables 5,6. We also assume the viscoelastic parameters, for Jupiter and Saturn respectively, $G=\{4.85,4.45\} \times 10^{10}(\mathrm{~Pa})$, and $\eta=\{1.26,1.78\} \times 10^{14}(\mathrm{Pas})$.

- we then fixed the value of the shear modulus $G$ to the lowest value needed to reach the observed tidal dissipation of Lainey et al. (2009-2012), i.e. $G=4.85 \times 10^{10} \mathrm{~Pa}$ for Jupiter, and $G=4.45 \times 10^{10}$ Pa for Saturn;

- we finally searched the more realistic value of the viscosity which corresponds to the observed tidal dissipations of Jupiter and Saturn: $\eta=1.26 \times 10^{14} \mathrm{~Pa}$ s for Jupiter, and $\eta=1.78 \times 10^{14} \mathrm{~Pa}$ s for Saturn.

For the present model, Fig. 10 explores, the dependence of $\hat{Q}_{\text {eff }}$ on the pulsation $\omega$ and the size of the core $R_{\mathrm{c}}$ normalized by the size of the planet $R_{\mathrm{p}}$. With these parameters, the figure indicates that Saturn dissipates slightly greater than ten times more than Jupiter, since $\left(R_{\mathrm{c}}\right)_{\text {Saturn }}>\left(R_{\mathrm{c}}\right)_{\text {Jupiter }}$ and $\left(\rho_{\mathrm{c}}\right)_{\text {Saturn }}<\left(\rho_{\mathrm{c}}\right)_{\text {Jupiter }}$. For the range of tidal frequencies of Jupiter's and Saturn's satellites $\left(2.25 \times 10^{-4} \mathrm{rad} \mathrm{s}^{-1}<\omega<2.95 \times 10^{-4} \mathrm{rad} \mathrm{s}^{-1}\right.$, Lainey et al. 2009-2012), the effective dissipation factor $\hat{Q}_{\text {eff }}$ remains almost constant, although it strongly depends on the size of the core, decreasing up to six orders of magnitude between a coreless planet and a fully-solid one. One can note that for a given core (where $M_{\mathrm{c}}, R_{\mathrm{c}}$, and then $\rho_{\mathrm{c}}$ are fixed) and a given mass of the planet $M_{\mathrm{p}}$, the density of the fluid envelope $\rho_{\mathrm{o}}$, which varies with its height $R_{\mathrm{p}}-R_{\mathrm{c}}$, cannot exceed $\rho_{\mathrm{c}}$. Since

$\rho_{\mathrm{o}}\left(R_{\mathrm{c}}\right)=\frac{M_{\mathrm{p}}-M_{\mathrm{c}}}{4 / 3 \pi\left(R_{\mathrm{p}}^{3}-R_{\mathrm{c}}^{3}\right)}$,

this condition gives a limit to the core size of

$\left(\frac{R_{\mathrm{c}}}{R_{\mathrm{p}}}\right)_{\text {sup }}=\left(\frac{M_{\mathrm{c}}}{M_{\mathrm{p}}}\right)^{1 / 3}$.

In 2004, Ogilvie \& Lin also studied tidal dissipation in giant planets, particulary the tidal dissipation resulting from the excitation of inertial waves in the convective region by the tidal potential for rotating giant planets with an elastic solid core. They obtained a decrease in the quality factor $Q$ of one order of magnitude for the dynamical tide, caused by inertial modes, relative to the equilibrium one, from $Q=10^{6}$ to $Q=10^{5}$. This was not, however, efficient enough to explain the observed tidal dissipation in Jupiter or Saturn, which is of 1-2 orders of magnitude higher (Lainey et al. 2009-2012). Moreover, they showed that the dissipation resulting from the resonance between fluid tide and inertial modes depends strongly on the tidal frequency in the range of inertial waves, as in the case of the coreless models (Wu 2005). This disagrees with the weak frequency-dependence inferred from astrometry (Lainey et al. 2012).

By discussing the size of the core, Goodman \& Lackner (2009) found a higher quality factor $Q$ in the range $10^{7}-10^{8} \times$ $\left(0.2 R_{\mathrm{p}} / R_{\mathrm{c}}\right)^{5}$, which disagrees with the observed value of the tidal dissipation of Saturn (see Fig. 9).

The present two-layer model proposes an alternative process to reach such a high dissipation with a smooth frequencydependence of $\hat{Q}$. However, the uncertainties in the structural and rheological parameters do not allow us to firmly conclude that the tidal dissipation of the core can explain on its own the tidal dissipation observed in giant planets of our solar system by Lainey et al. (2009-2012).

On the basis of these expressions of the tidal dissipation, which are closely linked to the internal structure of the planet and its rheological properties, we are able to derive the equations of the dynamical evolution of the system in terms of its explicit dependence on the tidal frequency.

\subsection{Comparison with previous work of Dermott}

The difference between our study and Dermott (1979) is the treatment of tidal dissipation.

Dermott based his formulae on an evolution scenario of Saturnian and Jovian systems. He assumed that the satellites of Jupiter and Saturn were formed $4.5 \times 10^{9}$ ago, and that their semi-major axis have changed by $10 \%$ since their formation because of a old stable resonance between the main satellites of Jupiter (Io, Europa and Ganymede) but a young resonance between the satellites Mimas and Thetys of Saturn. Dermott also assumed an average value of the tidal dissipation which where independent of frequency and time. All these assumptions led Dermott (1979) to a tidal dissipation factor $Q$ that only depends on the mass $M_{\mathrm{c}}$, the size $R_{\mathrm{c}}$, the elasticity $\mu$ of the core, and a dimensionless coefficient $K$ that is characteristic of the evolution 


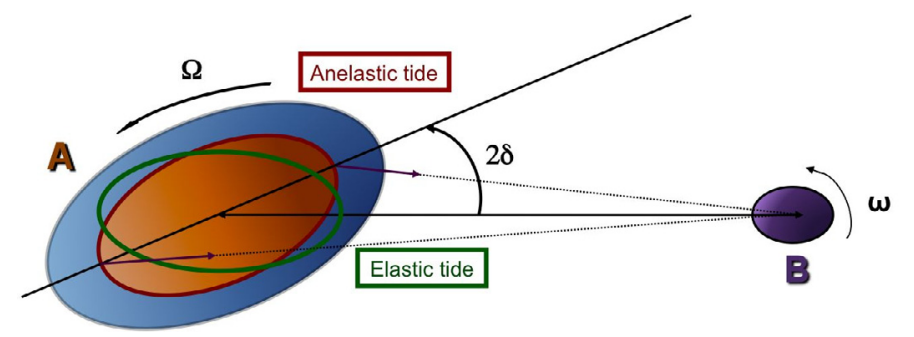

Fig. 11. Tidal interaction involving a solid body. Body $B$ exerts a tidal force on body $A$, which adjusts itself with a phase lag $2 \delta$, because of internal friction in the anelastic core. This adjustment may be separated into an adiabatic component, corresponding to the elastic deformation, that is in phase with the tide, and a dissipative one, resulting from the viscous internal frictions, which is in quadrature.

scenario of the planet. In particular, his tidal factor $Q$ is directly proportional to $R_{\mathrm{c}}^{5}$ (Eq. (27) of Dermott 1979), such that the tidal dissipation gets lower as the core size increases (see Fig. 4 of Dermott 1979), where one should expect an opposite behavior.

Our model is instead based on physical considerations of the internal structure and properties of the core. In particular, we derived our tidal dissipation factor $\hat{Q}$ with no assumption about the evolution of the Jovian and Saturnian systems. To do so, we used the correspondence principle of Biot (1954), which allowed us to obtain an expression for the tidal dissipation factor that is valid for any rheological model of planets' cores. Moreover, our expression in Eq. (82) for $\hat{Q}$ depends not only on the mass $M_{\mathrm{c}}$, size $R_{\mathrm{c}}$, and elasticity $G \equiv \mu$ of the core, but also on the tidal frequency $\omega$ and viscosity $\eta$. We note, in particular, that tidal dissipation increases when the size of the core increases, in contrast to Dermott's strange result.

\section{Equations of the dynamical evolution}

Mass redistribution due to tides generates in turn a tidal torque of a non-zero average that induces an exchange of angular momentum between the orbital motion and the rotation of each component. As shown in MLP09 \& Remus et al. (2012), this tidal torque is proportional to the tidal dissipation ratio $\left|\tilde{k}_{2}^{F}\right| / Q$ (see also Correia \& Laskar 2003; Correia et al. 2003; Murray \& Dermott 2000). One can note that for a perfectly elastic material, the core is elongated in the direction of the line of centers, inducing a torque, $\int_{\mathcal{V}} \boldsymbol{r} \wedge\left(\rho_{\mathrm{c}} \boldsymbol{\nabla} U\right) \mathrm{d} \mathcal{V}$, with periodic variations in the zero average, such that no secular exchanges of angular momentum are possible (see Zahn 1966a; Remus et al. 2012). However, if the core is anelastic, the deformation of the core resulting from the equilibrium adjustment presents a time delay $\Delta t$ with respect to the tidal forcing, which may also be measured by the tidal lag angle $2 \delta_{l}$ or equivalently by the quality factor $Q$ (see Ferraz-Mello et al. 2008; or Efroimsky \& Williams 2009)

$\sin \left[\Delta t \times \sigma_{2, m, p, q}\right]=\sin \left[2 \delta\left(\sigma_{2, m, p, q}\right)\right]=\frac{1}{Q\left(\sigma_{2, m, p, q}\right)}$.

Thus, the tidal bulge is no more aligned with the line of centers, as shown in Fig. 11.

The resulting tidal angle produces a torque of non-zero average that in turn causes an exchange of spin and orbital angular momentum between the components of the system.

The evolution of the semi-major axis $a$, the eccentricity $e$, the inclination $I$, the obliquity $\varepsilon$, and the angular velocity $\Omega$ (where $\bar{I}_{A}$ denotes the moment of inertia of $A$ ), is governed by equations, established in MLP09 and Remus et al. (2012),

$$
\begin{aligned}
& \frac{\mathrm{d}\left(\bar{I}_{\mathrm{A}} \Omega\right)}{\mathrm{d} t}=-\frac{8 \pi}{5} \frac{G M_{B}^{2} R_{\mathrm{eq}}^{5}}{a^{6}} \\
& \times \sum_{(m, j, p, q) \in \mathbb{I}}\left\{\frac{\left|\tilde{k}_{2}^{F}\left(R_{\mathrm{p}}, \sigma_{2, m, p, q}\right)\right|}{\hat{Q}_{\mathrm{eff}}\left(\sigma_{2, m, p, q}\right)}\left[\mathcal{H}_{2, m, j, p, q}(e, I, \varepsilon)\right]^{2}\right\}, \\
& \bar{I}_{A} \Omega \frac{\mathrm{d}(\cos \varepsilon)}{\mathrm{d} t}=\frac{4 \pi}{5} \frac{\mathcal{G} M_{B}^{2} R_{\mathrm{eq}}^{5}}{a^{6}} \\
& \times \sum_{(m, j, p, q) \in \mathbb{I}}\left\{(j+2 \cos \varepsilon) \frac{\left|\tilde{k}_{2}^{F}\left(R_{\mathrm{p}}, \sigma_{2, m, p, q}\right)\right|}{\hat{Q}_{\mathrm{eff}}\left(\sigma_{2, m, p, q}\right)}\left[\mathcal{H}_{2, m, j, p, q}(e, I, \varepsilon)\right]^{2}\right\}, \\
& \frac{1}{a} \frac{\mathrm{d} a}{\mathrm{~d} t}=-\frac{2}{n} \frac{4 \pi}{5} \frac{\mathcal{G} M_{B} R_{\mathrm{eq}}^{5}}{a^{8}} \\
& \times \sum_{(m, j, p, q) \in \mathbb{I}}\left\{(2-2 p+q) \frac{\left|\tilde{k}_{2}^{F}\left(R_{\mathrm{p}}, \sigma_{2, m, p, q}\right)\right|}{\hat{Q}_{\mathrm{eff}}\left(\sigma_{2, m, p, q}\right)}\left[\mathcal{H}_{2, m, j, p, q}(e, I, \varepsilon)\right]^{2}\right\}, \\
& \frac{1}{e} \frac{\mathrm{d} e}{\mathrm{~d} t}=-\frac{1}{n} \frac{1-\mathrm{e}^{2}}{\mathrm{e}^{2}} \frac{4 \pi}{5} \frac{\mathcal{G} M_{B} R_{\mathrm{eq}}^{5}}{a^{8}} \\
& \times \sum_{(m, j, p, q) \in \mathbb{I}}\left\{\left[(2-2 p)\left(1-\frac{1}{\sqrt{1-\mathrm{e}^{2}}}\right)+q\right]\right. \\
& \left.\times \frac{\left|\tilde{k}_{2}^{F}\left(R_{\mathrm{p}}, \sigma_{2, m, p, q}\right)\right|}{\hat{Q}_{\mathrm{eff}}\left(\sigma_{2, m, p, q}\right)}\left[\mathcal{H}_{2, m, j, p, q}(e, I, \varepsilon)\right]^{2}\right\}, \\
& \frac{\mathrm{d}(\cos I)}{\mathrm{d} t}=\frac{1}{n} \frac{1}{\sqrt{1-\mathrm{e}^{2}}} \frac{4 \pi}{5} \frac{G M_{B}^{2} R_{\mathrm{eq}}^{5}}{a^{8}} \\
& \times \sum_{(m, j, p, q) \in \mathbb{I}}\{[j+(2 q-2) \cos I] \\
& \left.\times \frac{\left|\tilde{k}_{2}^{F}\left(R_{\mathrm{p}}, \sigma_{2, m, p, q}\right)\right|}{\hat{Q}_{\mathrm{eff}}\left(\sigma_{2, m, p, q}\right)}\left[\mathcal{H}_{2, m, j, p, q}(e, I, \varepsilon)\right]^{2}\right\},
\end{aligned}
$$

where the functions $\mathcal{H}_{m, j, p, q}(e, I, \varepsilon)$ are expressed in terms of $d_{j, m}^{2}(\varepsilon), F_{2, j, p}(I)$, and $G_{2, p, q}(e)$, which are defined in Sect. 2.2

$$
\begin{aligned}
\mathcal{H}_{2, m, j, p, q}(e, I, \varepsilon)= & \sqrt{\frac{5}{4 \pi} \frac{(2-|j|) !}{(2+|j|) !}} \\
& \times d_{j, m}^{2}(\varepsilon) F_{2, j, p}(I) G_{2, p, q}(e),
\end{aligned}
$$

and $R_{\text {eq }}$ designates the equatorial radius of body $A$.

From these equations, one may derive the characteristic times of synchronization, circularization, and spin alignment

$$
\begin{aligned}
& \frac{1}{t_{\text {sync }}}=-\frac{1}{\Omega-n} \frac{\mathrm{d} \Omega}{\mathrm{d} t}=-\frac{1}{\bar{I}_{A}(\Omega-n)} \frac{\mathrm{d}\left(\bar{I}_{A} \Omega\right)}{\mathrm{d} t}, \\
& \frac{1}{t_{\text {circ }}}=-\frac{1}{e} \frac{\mathrm{d} e}{\mathrm{~d} t} \\
& \frac{1}{t_{\text {align }}}=-\frac{1}{\varepsilon} \frac{\mathrm{d} \varepsilon}{\mathrm{d} t}=\frac{1}{\varepsilon \sin \varepsilon} \frac{\mathrm{d}(\cos \varepsilon)}{\mathrm{d} t} \\
& \frac{1}{t_{\text {align }}}=-\frac{1}{I} \frac{\mathrm{d} I}{\mathrm{~d} t}=\frac{1}{I \sin I} \frac{\mathrm{d}(\cos I)}{\mathrm{d} t}
\end{aligned}
$$




\section{Conclusion}

We have studied the tidal dissipation in a two-layer planet consisting in a rocky/icy core and a fluid envelope, as one expects to be the case in Jupiter, Saturn, and many extrasolar planets. We have considered the most general configuration, where the perturber (star or satellite) moves on an elliptical and inclined orbit around the planet that rotates about an inclined axis. We have expanded the tidal displacement in the Fourier series and spherical harmonics, each term of the expansion having a radial part that is proportional to the corresponding term of the tidal potential, which depends on the eccentricity, inclination and obliquity. We followed the method of Dermott (1979) to derive the modified Love numbers $h_{2}^{F}$ and $k_{2}^{F}$ accounting for the tidal deformation at the boundary of the solid core. As in Dermott, we made the simplifying assumption that the core and envelope have a constant density. Then, generalizing the results of his work invoking the correspondence principle, we obtained the tidal dissipation rate of the core expressed by $\left|k_{2}^{F}\right| / \hat{Q}$, where $\hat{Q}$ is the quality factor. This ratio depends on the tidal frequency and the rheological properties of the core; unlike Dermott, we made no assumption about the formation history of the system. As mentioned in Sect. 5.1 the rheological properties of planetary cores are still quite uncertain. However, taking plausible values for the viscoelastic parameters $G$ and $\eta$, we obtained a tidal dissipation that may be much higher than for a fully fluid planet and weakly frequency-dependent. Under these assumptions, we found that the low value of $Q=(1.682 \pm 0.540) \times 10^{3}$, determined by Lainey et al. (2012) and needed by Charnoz et al. (2011) to explain the formation of all mid-sized moons of Saturn from the rings, can be reached by taking into account the tidal dissipation of Saturn's core. In the same way, the dissipation in Jupiter's core may explain the value of the $Q$-factor determined by Lainey et al. $(2009)$, i.e. $Q=(3.56 \pm 0.56) \times 10^{4}$. However, to do so, we need to assume a core in Jupiter and Saturn that is slightly larger than the values resulting from the models of Guillot (1999) and Hubbard et al. (2009). In our model, we recall that the density was assumed to be piecewise constant. In the future, we will consider a non-constant density profile, to evaluate the impact on our results of a realistic density stratification. Moreover, there are many uncertainties in the determination of the core sizes of giant planets such as Jupiter and Saturn, hence we need more constraints on the system formation by core accretion (Pollack et al. 1996) and differenciation resulting from the internal structure evolution (Nettelmann 2011). Furthermore, seismology seems to offer an interesting way of improving our knowledge of giant-planet interiors (Gaulme et al. 2011).

To conclude, we have studied tidal dissipation in the solid parts of a simple model of a two-layer planet, and illustrated the power of this mechanism. The results derived here are general in the sense that no specific rheological model has been assumed. However, owing to the lack of constraints on the rheology of giant planet cores, we have chosen the simplest Maxwell model to illustrate the tidal dissipation.

This work represents thus a first step toward more detailed numerical investigations of more realistic cases.

Acknowledgements. The authors are grateful to the referee for his/her remarks and suggestions. They also thank G. Tobie for fruitful discussions during this work and T. Guillot for providing numerical models of Jupiter and Saturn interiors. This work was supported in part by the Programme National de Planétologie (CNRS/INSU), the EMERGENCE-UPMC project EME0911, and the CNRS programme Physique théorique et ses interfaces.

\section{Appendix: elastic system}

In Sect. 2.3, we gave the system of Eq. (11), with its boundary conditions given in Eq. (12), governing an elastic planet under tidal perturbation. Since these perturbations are of small order of magnitude compared to the hydrostatic equilibrium, we proposed in Sect. 2.4 a method to linearize the system of Eq. (14). Thus, assuming the expansion in Eq. (18) of all quantities in spherical harmonics, we obtain the system of equations governing the scalar radial parts of these expansions (Alterman et al. 1959; Takeuchi \& Saito 1972)

$$
\begin{aligned}
\dot{y}_{1}^{m}= & -\frac{2\left(K-\frac{2}{3} \mu\right)}{K+\frac{4}{3} \mu} \frac{y_{1}^{m}}{r}+\frac{1}{K+\frac{4}{3} \mu} y_{2}^{m}+\frac{6\left(K-\frac{2}{3} \mu\right)}{K+\frac{4}{3} \mu} \frac{y_{3}^{m}}{r}, \\
\dot{y}_{2}^{m}= & {\left[-4 \rho_{0} g_{s} \frac{r}{R}+\frac{12 \mu K}{\left(K+\frac{4}{3} \mu\right) r}\right] \frac{y_{1}^{m}}{r}-\frac{4 \mu K}{\left(K+\frac{4}{3} \mu\right) r} \frac{y_{2}^{m}}{r} } \\
& +6\left[\rho_{0} g_{s} \frac{r}{R}-\frac{6 \mu K}{\left(K+\frac{4}{3} \mu\right) r}\right] \frac{y_{3}^{m}}{r}+6 \frac{y_{4}^{m}}{r}-\rho_{0} y_{6}^{m}, \\
\dot{y}_{3}^{m}= & -\frac{y_{1}^{m}}{r}+\frac{y_{3}^{m}}{r}+\frac{y_{4}^{m}}{\mu}, \\
\dot{y}_{4}^{m}= & {\left[\rho_{0} g_{s} \frac{r}{R}-\frac{6 \mu K}{\left(K+\frac{4}{3} \mu\right) r}\right] \frac{y_{1}^{m}}{r} } \\
& +\frac{2 \mu\left[11\left(K-\frac{2}{3} \mu\right)+10 \mu\right]}{\left(K+\frac{4}{3} \mu\right) r} \frac{y_{3}^{m}}{r}, \\
\dot{y}_{5}^{m}= & 4 \pi G \rho_{0} y_{1}^{m}+y_{6}^{m}, \\
\dot{y}_{6}^{m}= & -24 \pi G \rho_{0} \frac{y_{3}^{m}}{r}+\frac{6}{r} \frac{y_{5}^{m}}{r}-2 \frac{y_{6}^{m}}{r} .
\end{aligned}
$$

Solutions of Eq. (A.109) are given by Eq. (19).

\section{References}

Alterman, Z., Jarosch, H., \& Pekeris, C. L. 1959, Roy. Soc. London Proc. Ser. A, 252, 80

Biot, M. A. 1954, J. Appl. Phys., 25, 1385

Charnoz, S., Crida, A., Castillo-Rogez, J. C., et al. 2011, Icarus, 216, 535

Charette, M. A., \& Smith, W. H. F. 2010, Oceanography, 104, 106

Chree, C. 1896, Cambridge Phil. Trans., 16, 14

Correia, A. C. M., \& Laskar, J. 2003, Icarus, 163, 24

Correia, A. C. M., Laskar, J., \& de Surgy, O. N. 2003, Icarus, 163, 1

Dahlen, F. A., Tromp, J., \& Lay, T. 1999, Physics Today, 52, 61

Dermott, S. F. 1979, Icarus, 37, 310

Efroimsky, M., \& Williams, J. G. 2009, Cel. Mech. Dyn. Astron., 104, 257

Ferraz-Mello, S., Rodriguez, A., \& Hussmann, H. 2008, Cel. Mech. Dyn. Astron., 101, 171

Gaulme, P., Schmider, F.-X., Gay, J., Guillot, T., \& Jacob, C. 2011, A\&A, 531, A104

Gavrilov, S. V., \& Zharkov, V. N. 1977, Icarus, 32, 443

Goodman, J., \& Lackner, C. 2009, ApJ, 696, 2054

Greff-Lefftz, M., Métivier, L., \& Legros, H. 2005, Cel. Mech. Dyn. Astron., 93, 113

Guillot, T. 1999, Planet. Space Sci., 47, 1183

Guillot, T. 2005, Ann. Rev. Earth Planet. Sci., 33, 493

Guillot, T., \& Morel, P. 1995, A\&AS, 109, 109

Guillot, T., Chabrier, G., Gautier, D., \& Morel, P. 1995, ApJ, 450, 463

Henning, W. G., O'Connell, R. J., \& Sasselov, D. D. 2009, ApJ, 707, 1000

Hubbard, W. B., Dougherty, M. K., Gautier, D., \& Jacobson, R. 2009, Saturn from Cassini-Huygens, 75

Karato, S.-I. 2011, Icarus, 212, 14

Kaula, W. M. 1962, AJ, 67, 300

Lainey, V., Arlot, J.-E., Karatekin, Ö., \& van Hoolst, T. 2009, Nature, 459, 957 
F. Remus et al.: Anelastic tidal dissipation in multi-layer planets

Lainey, V., Karatekin, Ö., Desmars, J., et al. 2012, ApJ, in press [arXiv: 1204.0895]

Lambeck, K. 1980, The Earth's Variable Rotation (Cambridge University Press)

Love, A. E. H. 1911, Some Problems of Geodynamics (Cambridge University Press)

Mathis, S., \& Le Poncin-Lafitte, C. 2009, A\&A, 497, 889

Mathis, S., \& Zahn, J.-P. 2005, A\&A, 440, 653

Melchior, P. 1966, The Earth Tides (New York: Pergamon)

Militzer, B., \& Hubbard, W. B. 2009, Ap\&SS, 322, 129

Militzer, B., Hubbard, W. B., Vorberger, J., Tamblyn, I., \& Bonev, S. A. 2008, ApJ, 688, L45

Murray, C. D., \& Dermott, S. F. 2000, Solar system Dynamics (Cambridge University Press)

Nettelmann, N. 2011, Ap\&SS, 336, 47

Nettelmann, N., Holst, B., Kietzmann, A., et al. 2008, ApJ, 683, 1217

Ogilvie, G. I. 2009, MNRAS, 396, 794

Ogilvie, G. I., \& Lin, D. N. C. 2004, ApJ, 610, 477

Ogilvie, G. I., \& Lin, D. N. C. 2007, ApJ, 661, 1180

Peale, S. J., \& Cassen, P. 1978, Icarus, 36, 245
Pollack, J. B., Hubickyj, O., Bodenheimer, P., et al. 1996, Icarus, 124, 62

Remus, F., Mathis, S., \& Zahn, J.-P. 2012, A\&A, accepted

Rieutord, M. 1987, Geophysical and Astrophysical Fluid Dynamics, 39, 163

Ross, M., \& Schubert, G. 1986, Lunar and Planetary Science Conference Proceedings, 16, 447

Santos, N. C., et al. 2007, JENAM-2007, Our Non-Stable Universe

Saumon, D., \& Guillot, T. 2004, ApJ, 609, 1170

Takeuchi, H., \& Saito, M. 1972, Seismic surface waves, Methods Comput. Phys., 11,217

Thomson, W. (Lord Kelvin) 1863, Dynamical problems regarding elastic spheroidal shells, and On the rigidity of the Earth, Phil. Trans. Roy. Soc. London, 153, 573

Tobie, G. 2003, Ph.D. Thesis, Université Paris 7 - Denis Diderot

Tobie, G., Mocquet, A., \& Sotin, C. 2005, Icarus, 177, 534

Wu, Y. 2005, ApJ, 635, 688

Yoder, C. F. 1995, Icarus, 117, 250

Zahn, J.-P. 1966a, Annales d'Astrophysique, 29, 313

Zahn, J. P. 1966b, Annales d'Astrophysique, 29, 489

Zahn, J.-P. 1977, A\&A, 57, 383 